\title{
Effective Field Theory of Emergent Symmetry Breaking in Deformed Atomic Nuclei
}

\author{
T. Papenbrock ${ }^{1,2}$ and H. A. Weidenmüller ${ }^{3}$ \\ ${ }^{1}$ Department of Physics and Astronomy, University of Tennessee, Knoxville, TN \\ 37996, USA \\ ${ }^{2}$ Physics Division, Oak Ridge National Laboratory, Oak Ridge, TN 37831 USA \\ ${ }^{3}$ Max-Planck Institut für Kernphysik, D-69029 Heidelberg, Germany \\ E-mail: tpapenbr@utk.edu, haw@mpi-hd.mpg.de
}

\begin{abstract}
Spontaneous symmetry breaking in non-relativistic quantum systems has previously been addressed in the framework of effective field theory. Low-lying excitations are constructed from Nambu-Goldstone modes using symmetry arguments only. We extend that approach to finite systems. The approach is very general. To be specific, however, we consider atomic nuclei with intrinsically deformed ground states. The emergent symmetry breaking in such systems requires the introduction of additional degrees of freedom on top of the Nambu-Goldstone modes. Symmetry arguments suffice to construct the low-lying states of the system. In deformed nuclei these are vibrational modes each of which serves as band head of a rotational band.
\end{abstract}




\section{Introduction}

In this paper we present a detailed approach towards emergent symmetry breaking in finite non-relativistic quantum systems that uses concepts of effective field theory (EFT) [1, 2, 3]. A short summary of this approach was previously presented in Ref. [4], but without any details. Spontaneous symmetry breaking and the use of EFT in infinitely extended non-relativistic quantum systems such as ferromagnets is well established [5, 6]. Our work is specifically tailored for finite systems. Examples are finite Bose-Einstein condensates or BCS superconductors with a broken $U(1)$ phase symmetry and molecules and nuclei that possess deformed ground states and, thus, break

rotational invariance. In finite systems there is no spontaneous symmetry breaking in the strict sense, and we speak instead of emergent symmetry breaking [7]. The description of such systems within an EFT requires, aside from the standard NambuGoldstone modes, additional degrees of freedom. To be specific we address here the case of deformed nuclei. The additional degrees of freedom then describe rotations of the entire nucleus. The treatment of emergent symmetry breaking in other systems requires only minor modifications, however.

To set the stage we recall some aspects of the theory of nuclei with deformed ground states such as occur in rare-earth nuclei and in the actinides. Low-lying excitations are traditionally described phenomenologically as rotations and vibrations, both in the collective geometric model [8] and in the algebraic model [9]. Both models describe certain "leading order" aspects (or gross features) very well: Low-lying excitations are vibrational states that serve as band heads of rotational bands. Electromagnetic intraband transitions are very strong, inter-band transitions are much weaker. These models typically fail to account quantitatively for finer details regarding "next-to-leading order" effects such as the change of the moment of inertia with the vibrational band head or the magnitude of weak E2 inter-band transitions. (Both deficiencies can be addressed within an effective theory [10, 11].)

In this paper we take the view that nuclear ground-state deformation (and, incidentally, pairing) are emergent phenomena. That view is supported by first $a b$ initio calculations of rotational bands in light nuclei [12, 13]. Similarly, nuclear meanfield calculations yield microscopic evidence for ground-state deformations [14]. For such microscopic approaches the description of emergent phenomena is a challenge, however, because they present multi-scale problems. Therefore, the traditional approach within the Wigner-Weyl (linear) realization of the underlying symmetry requires very large model spaces to describe the emergent symmetry breaking.

The approach using an EFT is based entirely upon symmetry arguments. The emergent broken symmetry is treated using the nonlinear Nambu-Goldstone realization of the symmetry (as opposed to the linear Wigner-Weyl realization) plus those additional degrees of freedom that account for rotations. The combined treatment of these degrees of freedom is the novel technical aspect of our work. The approach has the advantage of being model independent. A controlled expansion in terms of well-defined small 
parameters generates higher-order terms is a systematic fashion. These may serve as useful guides in the phenomenological models.

Aside from presenting details of the EFT approach outlined in Ref. [4], it is the purpose of this paper to show how such higher-order terms are generated and how they influence results of lower order. Moreover we establish the connection of our EFT with the technically simpler effective theory of deformed nuclei developed in Ref. [15]. Our results show that the effective theory is based upon a solid field-theoretical foundation.

\section{General Approach}

We consider a deformed liquid drop with a space-fixed center of mass and with axial symmetry about the body-fixed $z^{\prime}$-axis. In the present Section we introduce the NambuGoldstone modes and in addition the time-dependent modes that describe rotational motion. We warn the reader ahead of time that in Section 3 we switch to another parameterization of these modes. The parameterization used in the present Section is physically transparent and easy to justify. It has the drawback that it leads to analytically cumbersome expressions. The parameterization used in Section 3 is easy to handle but based on a different physical picture. In order to exhibit the general scheme we follow the approach of the present Section up to the construction of the classical field theory and its symmetries. The developments of Section 3 then run in parallel to these developments.

\subsection{Nambu-Goldstone Modes}

We denote the Cartesian coordinates in the space-fixed system $S$ (the body-fixed system $S^{\prime}$ ) by $\{x, y, z\}$ (by $\left\{x^{\prime}, y^{\prime}, z^{\prime}\right\}$, respectively). The nuclear ground state is invariant under $S O(2)$ rotations about the body-fixed $z^{\prime}$-axis while $S O(3)$ symmetry is broken by the deformation. The Nambu-Goldstone modes parameterize the two-dimensional coset space $S O(3) / S O(2)$ and depend on two complex fields $\pi_{ \pm}$. In the body-fixed system, a mass element of the liquid drop has spherical coordinates $(r, \theta, \phi)$. The classical fields $\pi_{ \pm}$depend on these dynamical variables and on time $t$. We neglect the $r$-dependence of $\pi_{ \pm}$because radial vibrations of the liquid drop are expected to have higher excitation energies than surface vibrations. We denote the remaining coordinates $\theta, \phi$ and time $t$ jointly by $\rho_{\mu}$ with $\mu=1,2,3$. The fields $\pi_{ \pm}$generate the local rotation

$$
U(\rho)=\exp \left\{-i \pi_{-}(\rho) P_{+}-i \pi_{+}(\rho) P_{-}\right\}
$$

We follow Ref. [16] and denote the total angular momentum operator in the body-fixed system by $\vec{P}$ and in the space-fixed system by $\vec{J}$. Then $P_{x^{\prime}}, P_{y^{\prime}}, P_{z^{\prime}}$ are the components of $\vec{P}$ in the body-fixed system, and

$$
P_{ \pm}=\mp \frac{1}{\sqrt{2}}\left(P_{x^{\prime}} \pm i P_{y^{\prime}}\right)
$$

Although we use the expression "angular momentum operator" for $\vec{P}$ and $\vec{J}$, the three components of these operators represent in the present context only the three generators 
of rotations in the body-fixed and in the space-fixed system, respectively. With $\pi_{+}=\pi_{-}^{*}$, the transformation $U$ is unitary. For a physical interpretation of the fields $\pi_{ \pm}$we write

$$
\pi_{ \pm}=\frac{1}{2} \omega \exp \{ \pm i \zeta\}
$$

with $\omega$ and $\zeta$ as the new real dynamical fields so that Eq. (11) becomes

$$
U=\exp \left\{-i \omega\left[\cos \zeta P_{x^{\prime}}+\sin \zeta P_{y^{\prime}}\right]\right\} .
$$

We interpret Eq. (4) geometrically. In the body-fixed system, rotations are around an axis perpendicular to the symmetry axis. The azimuthal angle of the rotation axis is denoted as $\zeta$. The angle $\omega$ is the angle of rotation about that axis. Replacing $\zeta$ by $\zeta+\pi$ we change the orientation of the axis of rotation or, equivalently, the direction of rotation (clockwise rotation $\rightarrow$ counter-clockwise rotation). Clockwise rotation by the angle $\omega$ is equivalent to counter-clockwise rotation by the angle $2 \pi-\omega$. Therefore, we might confine the angle $\omega$ to $0 \leq \omega \leq \pi$. For reasons that will become apparent later we do not adopt that choice here so that the ranges of the angles $\zeta$ and $\omega$ are $0 \leq \omega, \zeta \leq 2 \pi$. We expect the effective Lagrangian to depend on trigonometric functions of $\omega$ and $\zeta$ only. The fields $\omega$ and $\zeta$ are functions of the dynamical variables $\rho_{\mu}, \mu=1,2,3$. Inspection shows that a pure time dependence of $\omega$ and $\zeta$ describes the rotation of the deformed nucleus as a whole while a genuine dependence of $\omega$ and $\zeta$ on the angles $\theta$ and $\phi$ accounts for surface vibrations, i.e., local dislocations of the constituents of the deformed drop (fluid elements or nucleons, as the case may be). Therefore, we write

$$
\begin{aligned}
& \omega=\omega_{0}(t)+\omega_{1}(\theta, \phi, t), \\
& \zeta=\zeta_{0}(t)+\zeta_{1}(\theta, \phi, t),
\end{aligned}
$$

with the understanding that $\omega_{1}$ and $\zeta_{1}$ possess a non-trivial dependence on at least one of the angles $\theta, \phi$ (so that not all partial derivatives with respect to these angles vanish identically). We expect (and verify later) that the functions $\omega_{1}$ and $\zeta_{1}$ define the true Nambu-Goldstone modes of the deformed nucleus that describe surface vibrations. For these we will use a small-amplitude approximation. The purely time-dependent functions $\omega_{0}(t)$ and $\zeta_{0}(t)$, on the other hand, are alien to the standard approach to spontaneously broken symmetry. They represent the novel element that accounts for the finite size of the system and describe rotational motion. For these functions we cannot use the small-amplitude approximation. This situation is technically similar to finite-volume EFTs of quantum chromodynamics [17, 18] and magnets [19]. Here - and in contrast to the genuine finite systems we consider - the finite volume stems from limitations of computational resources and obscures the physics of the infinite system one is interested in. In both cases, the purely time-dpendent "zero mode" exhibits large fluctuations and has to be treated separately.

We follow Ref. [5] and define the Nambu-Goldstone modes $a_{\mu}^{ \pm}$and $a_{\mu}^{z^{\prime}}$ of our problem by writing

$$
U^{\dagger}(\rho) i \partial_{\mu} U(\rho)=a_{\mu}^{-} P_{+}+a_{\mu}^{+} P_{-}+a_{\mu}^{z^{\prime}} P_{z^{\prime}}
$$


Here $\partial_{\mu}=\partial / \partial \rho_{\mu}$ for $\mu=1,2,3$. The coefficients $a_{\mu}^{ \pm}$and $a_{\mu}^{z^{\prime}}$ may alternatively be written as functions of the fields $\pi_{ \pm}$or of the fields $\omega, \zeta$. The effective Lagrangian is obtained by forming combinations of these coefficients that are invariant both with respect to the group operations and with respect to space rotations.

We express the Nambu-Goldstone modes in terms of $\omega$ and $\zeta$. The calculation is lengthy but straightforward. We use the left-hand side of Eq. (6), the form (4) for $U$, and a Taylor expansion for $U$. We find

$$
\begin{aligned}
& a_{\mu}^{-}=(1 / 2) \exp \{-i \zeta\}\left[\partial_{\mu} \omega-i\left(\partial_{\mu} \zeta\right) \sin \omega\right], \\
& a_{\mu}^{+}=(1 / 2) \exp \{+i \zeta\}\left[\partial_{\mu} \omega+i\left(\partial_{\mu} \zeta\right) \sin \omega\right] \\
& a_{\mu}^{z^{\prime}}=\left(\partial_{\mu} \zeta\right)(1-\cos \omega) .
\end{aligned}
$$

\subsection{Invariants}

To determine the behavior of $a_{\mu}^{ \pm}$and $a_{\mu}^{z^{\prime}}$ under group operations we consider the action of a fixed element $g$ (independent of the $\rho_{\mu}$ ) of the coset space on $U$. Equation (1) implies that under the action of $g, U$ changes nonlinearly,

$$
U \rightarrow[g U] h^{\dagger}(g, U) .
$$

The product $[g U]$ lies within the coset space $S O(3) / S O(2)$ while

$$
h(g, U)=\exp \left\{i \Psi(g, U) P_{z^{\prime}}\right\}
$$

is an element of $S O(2)$. The function $\Psi$ depends on the parameters characterizing both $g$ and the transformation $U$. Thus,

$$
U^{\dagger}(\rho) i \partial_{\mu} U(\rho) \rightarrow h U^{\dagger}(\rho) i\left[\partial_{\mu} U(\rho)\right] h^{\dagger}+\partial_{\mu} \Psi(g, U) P_{z^{\prime}}
$$

and

$$
a_{\mu}^{-} P_{+}+a_{\mu}^{+} P_{-}+a_{\mu}^{z^{\prime}} P_{z^{\prime}} \rightarrow h\left(a_{\mu}^{-} P_{+}+a_{\mu}^{+} P_{-}+a_{\mu}^{z^{\prime}} P_{z^{\prime}}\right) h^{\dagger}+\partial_{\mu} \Psi(g, U) P_{z^{\prime}}
$$

We recall the commutation relations

$$
\begin{aligned}
& {\left[J_{x}, J_{y}\right]=i J_{z} \text { (cyclic), }} \\
& {\left[P_{x^{\prime}}, P_{y^{\prime}}\right]=-i P_{z^{\prime}}(\text { cyclic }),} \\
& {\left[J_{k}, P_{l^{\prime}}\right]=0(\text { all } k, l=x, y, z) .}
\end{aligned}
$$

which imply

$$
\begin{aligned}
& {\left[J_{z}, J_{+}\right]=J_{+},\left[J_{z}, J_{-}\right]=-J_{-},\left[J_{+}, J_{-}\right]=-J_{z}} \\
& {\left[P_{z^{\prime}}, P_{+}\right]=-P_{+},\left[P_{z^{\prime}}, P_{-}\right]=P_{-},\left[P_{+}, P_{-}\right]=P_{z^{\prime}}}
\end{aligned}
$$

and $P_{z^{\prime}}^{m} P_{ \pm}=P_{ \pm}\left(P_{z^{\prime}} \mp 1\right)^{m}$. To calculate the right-hand side of expression (11) we use these for $\vec{P}$ and the expansion of $h$ in a Taylor series in $P_{z^{\prime}}$. We obtain

$$
\begin{aligned}
& a_{\mu}^{-} \rightarrow \exp \{-i \Psi\} a_{\mu}^{-}, \\
& a_{\mu}^{+} \rightarrow \exp \{i \Psi\} a_{\mu}^{+}, \\
& a_{\mu}^{z^{\prime}} \rightarrow a_{\mu}^{z^{\prime}}+\partial_{\mu} \Psi(g, U) .
\end{aligned}
$$


Therefore, the terms of lowest order in the $a_{\mu}^{ \pm}, a_{\mu}^{z^{\prime}}$ that are invariant under group operations are [5]

$$
a_{\mu}^{+} a_{\nu}^{-} \text {and } \partial_{\mu} a_{\nu}^{z^{\prime}}-\partial_{\nu} a_{\mu}^{z^{\prime}}
$$

Here $\mu, \nu=1,2,3$. The expressions (15) are obtained by treating the derivatives $\partial_{\mu}$ both with respect to the angles $\theta, \phi$ and with respect to time as though they were derivatives with respect to external variables. While that is appropriate for time $t(\mu=3)$, it is not for the angles $\theta, \phi(\mu=1,2)$ because these change under rotations. The effective Lagrangian must be invariant with respect to such rotations. In actually constructing the invariants we demand axial symmetry (see Section 3) and are guided by the analogy to coordinate transformations in Euclidean space in three dimensions. Here we would interpret $\partial_{k}$ with $k=x, y, z$ as one component of the vector $\vec{\nabla}$, use the invariance of the scalar product of two vectors, and obtain the invariants $\vec{\nabla} a^{+} \vec{\nabla} a^{-}$. On the unit sphere we analogously replace $\vec{\nabla}$ by the vector $\vec{L}$ of orbital angular momentum with components

$$
\begin{aligned}
L_{x^{\prime}} & =+i \sin \phi \frac{\partial}{\partial \theta}+i \cos \phi \cot \theta \frac{\partial}{\partial \phi} \\
L_{y^{\prime}} & =-i \cos \phi \frac{\partial}{\partial \theta}+i \sin \phi \cot \theta \frac{\partial}{\partial \phi} \\
L_{z^{\prime}} & =-i \frac{\partial}{\partial \phi}
\end{aligned}
$$

The lowest-order axially symmetric invariants formed from $a^{ \pm}$are then

$$
\begin{aligned}
& L_{x^{\prime}} a^{+} L_{x^{\prime}} a^{-}+L_{y^{\prime}} a^{+} L_{y^{\prime}} a^{-}, \\
& L_{z^{\prime}} a^{+} L_{z^{\prime}} a^{-}, \\
& \partial_{t} a^{+} \partial_{t} a^{-} .
\end{aligned}
$$

Expressions like $L_{x^{\prime}} a^{+}$are here understood as linear combinations of terms $a_{\mu}^{+}$(read as partial derivatives with respect to $\rho_{\mu}$ and explicitly given in Eqs. (7)) with coefficients given in the first of Eqs. (16). For simplicity, we employ in the present Section only the spherical invariant

$$
L_{x^{\prime}} a^{+} L_{x^{\prime}} a^{-}+L_{y^{\prime}} a^{+} L_{y^{\prime}} a^{-}+L_{z^{\prime}} a^{+} L_{z^{\prime}} a^{-},
$$

and use the more general axially symmetric invariants in Section 3 .

The substitution rules (14) apply in both the body-fixed and the space-fixed coordinate system, albeit with different definitions of the function $\Psi$. Using this fact and the well-known behavior of the components of $\vec{L}$ under rotations we easily confirm that the form (18) is indeed rotationally invariant.

The eigenstates of the nuclear Hamiltonian are (almost exactly) eigenstates of the parity operator. But that operator cannot be written in terms of rotations. Indeed, in three dimensions the determinant of the matrix describing parity inversion equals minus one while the determinants of all rotation matrices are equal to plus one because these connect continuously to the unit matrix. Therefore, we cannot incorporate parity 
conservation into the present approach. We return to that point in Section 4.2. The situation for time reversal differs. To be invariant under time reversal the effective Lagrangian must contain only even derivatives with respect to time. That constraint has been taken into account in expressions (17). The constraint is appropriate for eveneven nuclei with ground-state spin zero. Another discrete symmetry (the $\mathcal{R}$-parity of Ref. [8]) plays a role for the quantized version of the theory and is treated in Section 4.2. too.

The explicit form of the invariants is obtained by using Eqs. (7) in expressions (17) and (18),

$$
\begin{aligned}
& \mathcal{L}_{1}=2 a_{t}^{-} a_{t}^{+}=\frac{1}{2}\left(\dot{\omega}^{2}+\dot{\zeta}^{2} \sin ^{2} \omega\right) \\
& \mathcal{L}_{2}=2 \sum_{k=1}^{3}\left(L_{k} a^{+}\right)\left(L_{k} a^{-}\right)=\frac{1}{2}\left((\vec{L} \omega)^{2}+\sin ^{2} \omega(\vec{L} \zeta)^{2}\right) .
\end{aligned}
$$

The dot indicates the time derivative.

\subsection{Classical Field Theory}

The classical field theory is obtained by writing the effective Lagrangian density $\mathcal{L}$ as a linear combination of the two invariants (19),

$$
\mathcal{L}=C \mathcal{L}_{1}+D \mathcal{L}_{2}
$$

The coefficients $C$ and $D$ are the parameters of the theory and are determined by a fit to the data. The total Lagrangian $L$ is obtained by integrating $\mathcal{L}$ over the dynamical angles $\theta, \phi$,

$$
L=\int \mathrm{d} E \mathcal{L} \equiv \frac{1}{4 \pi} \int_{0}^{\pi} \mathrm{d} \theta \sin \theta \int_{0}^{2 \pi} \mathrm{d} \phi \mathcal{L} .
$$

The normalization is chosen such that for $\mathcal{L}=1$ the integral in Eq. (21) yields unity. The factor $\sin \theta$ in the measure (21) takes account of the fact that $\theta, \phi$ define points on the surface of a sphere so that we deal with curvilinear coordinates. We show in Appendix 1 that in such coordinates the equations of motion are

$$
\begin{gathered}
\sum_{\mu} \partial_{\mu}\left(\frac{\partial(\sin \theta \mathcal{L})}{\partial\left(\partial_{\mu} \omega\right)}\right)=\frac{\partial(\sin \theta \mathcal{L})}{\partial \omega}, \\
\sum_{\mu} \partial_{\mu}\left(\frac{\partial(\sin \theta \mathcal{L})}{\partial\left(\partial_{\mu} \zeta\right)}\right)=\frac{\partial(\sin \theta \mathcal{L})}{\partial \zeta} .
\end{gathered}
$$

We recall that $\mu=1,2$ stands for the angles $\theta, \phi$ and $\mu=3$ for the time $t$, and that both $\omega$ and $\zeta$ are functions of $\theta, \phi, t$. Eqs. (22), (20), and (19) constitute the non-linear equations of motion for the two classical fields $\omega$ and $\zeta$.

In constructing $\mathcal{L}$ we have imposed rotational invariance. Therefore, we expect that the total angular momentum of the system is conserved. Using the Noether theorem as in Ref. [15], we now construct three constants of the motion. These correspond to the 
three components of angular momentum (not to be confused with the three components of the rotation operator in Eqs. (12) ). With $k=x^{\prime}, y^{\prime}, z^{\prime}$ we consider the rotation

$$
r=\exp \left\{-i \sum_{k} \delta \chi_{k} P_{k}\right\}
$$

by infinitesimally small angles $\delta \chi_{k}$ about the three body-fixed axes. The ensuing infinitesimal changes of $\omega$ and of $\zeta$ are denoted by $\delta \omega$ and $\delta \zeta$, those of $\mathcal{L}$ by $\delta \mathcal{L}$. A straightforward calculation yields

$$
\delta \mathcal{L}=\sum_{k=1}^{3} \delta \chi_{k}\left\{\sum_{\mu=1}^{3} \frac{\partial}{\partial \mu}\left(\frac{\partial \mathcal{L}}{\partial\left(\partial_{\mu} \omega\right)} M_{1 k}+\frac{\partial \mathcal{L}}{\partial\left(\partial_{\mu} \zeta\right)} M_{2 k}\right)\right\} .
$$

With $\delta \omega=\delta q_{1}$ and $\delta \zeta=\delta q_{2}$ the matrix $M$ is defined by $\delta q_{\nu}=\sum_{k} M_{\nu k} \delta \chi_{k}$ and explicitly given in Appendix 2. Rotational invariance implies $\delta \mathcal{L}=0$ for every choice of $\delta \chi_{k}$ with $k=x^{\prime}, y^{\prime}, z^{\prime}$. That implies the vanishing of each of the three Noether currents $k=x^{\prime}, y^{\prime}, z^{\prime}$ in big curly brackets in Eq. (24).

To obtain the constants of the motion we consider an infinitesimal change of the total Lagrangian given by

$$
\delta L=\int \frac{\mathrm{d} \theta \mathrm{d} \phi}{4 \pi} \delta(\sin \theta \mathcal{L})
$$

with $\delta \mathcal{L}$ defined in Eq. (24). The effective Lagrangian density $\mathcal{L}$ is periodic in the angle $\phi$. With respect to $\theta$, the same statement holds for $\mathcal{L} \sin \theta$. Partial integration then shows that the derivative terms with respect to $\theta$ and $\phi$ in Eq. (24) do not contribute to $\delta L$ so that $\delta L$ is given by a pure time derivative. The vanishing of $\delta L$ for any choice of the angles $\delta \chi_{k}$ then implies the existence of three constants of the motion. These are the three components $Q_{k}$ of the total angular momentum of the system. With $M$ given in Eq. (118) of Appendix 2 and the integration measure defined in Eq. (21) these are

$$
\begin{aligned}
& Q_{x^{\prime}}=\int \mathrm{d} E\left(\frac{\partial \mathcal{L}}{\partial \dot{\omega}} \cos \zeta-\frac{\partial \mathcal{L}}{\partial \dot{\zeta}} \sin \zeta \cot \omega\right), \\
& Q_{y^{\prime}}=\int \mathrm{d} E\left(\frac{\partial \mathcal{L}}{\partial \dot{\omega}} \sin \zeta+\frac{\partial \mathcal{L}}{\partial \dot{\zeta}} \cos \zeta \cot \omega\right), \\
& Q_{z^{\prime}}=-\int \mathrm{d} E \frac{\partial \mathcal{L}}{\partial \dot{\zeta}} .
\end{aligned}
$$

\subsection{Power Counting}

Without much justification given, we have constructed the effective Lagrangian in Eqs. (20) and (19) from the lowest-order invariants in the Nambu-Goldstone modes. Further progress hinges on the identification of the energy scales that govern our problem and of the associated powers of higher-order terms in the Nambu-Goldstone modes. The relevant scales are the energy scale $\xi$ of rotational motion, the energy scale $\Omega$ of vibrational motion, and the cutoff parameter $\Lambda$ beyond which other modes like fermionic excitations play a role. In our approach it is assumed that $\xi \ll \Omega \ll \Lambda$. 
To identify the various contributions to the effective Lagrangian in Eq. (201) and to the equations of motion (22), we use the decomposition (5) and an expansion of $\omega_{1}$ and of $\zeta_{1}$ in terms of spherical harmonics,

$$
\begin{aligned}
\omega_{1} & =\sum_{L=2}^{\infty} \sum_{\mu} \omega_{L \mu}(t) Y_{L \mu}(\theta, \phi), \\
\zeta_{1} & =\sum_{L=2}^{\infty} \sum_{\mu} \zeta_{L \mu}(t) Y_{L \mu}(\theta, \phi) .
\end{aligned}
$$

The term with $L=1$ describes center-of-mass motion and is suppressed. As mentioned earlier we take rotational motion fully into account by treating $\omega_{0}$ and $\zeta_{0}$ without any approximation. We use a small-amplitude approximation for the surface vibrations described by $\omega_{1}$ and $\zeta_{1}$. A naive first approach would then consist in expanding $\mathcal{L}$ in powers of $\omega_{1}$ and of $\zeta_{1}$ and in keeping only terms up to second order (but terms of all orders in $\omega_{0}$ and in $\zeta_{0}$ ). That would be in line with the treatment of ferromagnets and paramagnets in Ref. [5] where in the leading-order effective Lagrangian only terms up to second order in the fields are kept. However, because of the presence of rotational motion that procedure does not fully apply in our case. To see that we must analyze the contributions to $\mathcal{L}$ in some detail. We use the symbol $\sim$ to define the relevant order, and we drop the magnetic quantum numbers on $\omega_{L}$ and $\zeta_{L}$.

The ratios $\dot{\omega}_{0} / \omega_{0} \sim \xi$ and $\dot{\zeta}_{0} / \zeta_{0} \sim \xi$ are governed by the energy scale of rotational motion. The range of the variables $\omega_{0}$ and $\zeta_{0}$ is of order unity. Therefore, $\dot{\omega}_{0} \sim \xi$ and $\dot{\zeta}_{0} \sim \xi$. Inserting Eqs. (5) into Eqs. (22) we obtain the terms $(C / 2) \dot{\omega}_{0}^{2}$ and $(C / 2) \dot{\zeta}_{0}^{2}$. We show below that these describe rotational motion. Therefore, $(C / 2) \dot{\omega}_{0}^{2} \sim \xi$ and $(C / 2) \dot{\zeta}_{0}^{2} \sim \xi$. Together with $\dot{\omega}_{0} \sim \xi$ and $\dot{\zeta}_{0} \sim \xi$ that implies $C \sim 1 / \xi$ which is consistent with the interpretation of $C$ as moment of inertia. The ratios $\dot{\omega}_{L} / \omega_{L} \sim \Omega$ and $\dot{\zeta}_{L} / \zeta_{L} \sim \Omega$ with $L \geq 2$ are similarly governed by the energy scale $\Omega$ of vibrational motion. Inserting Eqs. (5) into Eqs. (22) we obtain for $L \geq 2$ the terms $(C / 2) \dot{\omega}_{L}^{2}$ and $(C / 2) \dot{\zeta}_{L}^{2}$. These describe vibrational motion. Therefore $(C / 2) \dot{\omega}_{L}^{2} \sim \Omega$ and $(C / 2) \dot{\zeta}_{L}^{2} \sim \Omega$. Together with $C \sim 1 / \xi$ these relations imply $\dot{\omega}_{L}, \dot{\zeta}_{L} \sim \sqrt{\xi \Omega}$ and, together with $\dot{\omega}_{L} / \omega_{L} \sim \Omega, \dot{\zeta}_{L} / \zeta_{L} \sim \Omega$ also $\omega_{L} \sim \sqrt{\xi / \Omega}, \zeta_{L} \sim \sqrt{\xi / \Omega}$ for $L \geq 2$. These relations are used below when we expand $\mathcal{L}$ in powers of $\omega_{L}$ and $\zeta_{L}$. The terms in $\mathcal{L}_{2}$ (see Eqs. (19)) describe vibrational motion so we have $(D / 2)\left(\vec{L} \omega_{L}\right)^{2} \sim \Omega$ and $(D / 2)\left(\vec{L} \zeta_{L}\right)^{2} \sim \Omega$.

In our approach the operator $\vec{L}$ is dimensionless (see Eqs. (16)). Formally, however, $\vec{L}$ plays the same role as the momentum in the theory of Ref. [5] where only small momenta are kept for small energies. Physically we analogously expect that only the small eigenvalues of the operator $\vec{L}^{2}$ are relevant for the low-energy part of the spectrum in our case. Therefore we formally attach to $\vec{L}$ the scale $\Omega$. Together with $\omega_{L}, \zeta_{L} \sim \sqrt{\xi / \Omega}$ that gives $D \sim 1 / \xi$. We summarize these assumptions and results by writing

$$
\begin{aligned}
& \xi \quad \ll \Omega, \\
& \omega_{0}, \zeta_{0} \sim 1,
\end{aligned}
$$




$$
\begin{aligned}
& \omega_{L}, \zeta_{L} \sim \sqrt{\xi / \Omega} \ll 1 \text { for } L \geq 2, \\
& \dot{\omega}_{0}, \dot{\zeta}_{0} \sim \xi, \\
& \ddot{\omega}_{0}, \ddot{\zeta}_{0} \sim \xi^{2}, \\
& \dot{\omega}_{L}, \dot{\zeta}_{L} \sim \sqrt{\xi \Omega} \text { for } L \geq 2, \\
& \ddot{\omega}_{L}, \ddot{\zeta}_{L} \sim \Omega \sqrt{\xi \Omega} \text { for } L \geq 2, \\
& C \quad \sim \frac{1}{\xi}, \\
& D \quad \sim \frac{1}{\xi}, \\
& \vec{L} \quad \sim \Omega .
\end{aligned}
$$

These considerations imply the following rule for an approximate treatment of the problem. The effective Lagrangian $\mathcal{L}$ in Eqs. (20, 19) contains terms of order $\Omega$ that describe nuclear surface vibrations and terms of order $\xi$ that describe rotational motion. In expanding $\mathcal{L}$ in powers of $\omega_{L}$ and $\zeta_{L}$ we must, therefore, keep terms of orders $\Omega, \sqrt{\Omega \xi}$ and $\xi$. We omit terms of order $\sqrt{\xi / \Omega}$ or less. The resulting approximate expressions are

$$
\begin{aligned}
C \mathcal{L}_{1} \approx & \frac{C}{2}\left(\left(\dot{\omega}_{0}+\dot{\omega}_{1}\right)^{2}+\dot{\zeta}_{0}^{2} \sin ^{2} \omega_{0}\right. \\
& +2 \dot{\zeta}_{0} \dot{\zeta}_{1}\left[\sin ^{2} \omega_{0}+\omega_{1} \sin 2 \omega_{0}\right] \\
& \left.+\dot{\zeta}_{1}^{2}\left[\sin ^{2} \omega_{0}+\omega_{1} \sin 2 \omega_{0}+\omega_{1}^{2} \cos 2 \omega_{0}\right]\right), \\
D \mathcal{L}_{2} \approx & \frac{D}{2}\left((\vec{L} \omega)^{2}+(\vec{L} \zeta)^{2}\left[\sin ^{2} \omega_{0}+\omega_{1} \sin 2 \omega_{0}\right.\right. \\
& \left.\left.+\omega_{1}^{2} \cos 2 \omega_{0}\right]\right) .
\end{aligned}
$$

These developments show which terms to keep in the effective Lagrangian.

\subsection{Purely Rotational Motion}

To ascertain consistency of our arguments we consider the case of nuclear rotation without surface vibrations. We accordingly assume that $\omega$ and $\zeta$ depend only upon time, so that in Eqs. (5) we have $\omega_{1}=0=\zeta_{1}$. For purely time-dependent fields classical field theory changes into classical mechanics. The treatment becomes very similar to that of Ref. [15]. The effective Lagrangian is

$$
\mathcal{L}=\frac{C}{2}\left(\dot{\omega}_{0}^{2}+\dot{\zeta}_{0}^{2} \sin ^{2} \omega_{0}\right),
$$

and the canonical momenta are

$$
\begin{aligned}
& \pi_{\omega}=\frac{\partial \mathcal{L}}{\partial \dot{\omega}_{0}}=C \dot{\omega}_{0}, \\
& \pi_{\zeta}=\frac{\partial \mathcal{L}}{\partial \dot{\zeta}_{0}}=C \dot{\zeta}_{0} \sin ^{2} \omega_{0} .
\end{aligned}
$$


The Hamiltonian $H$ is given by

$$
H=\frac{1}{2 C}\left(\pi_{\omega}^{2}+\frac{1}{\sin ^{2} \omega_{0}} \pi_{\zeta}^{2}\right) .
$$

The three components $Q_{k}$ of angular momentum are given by Eqs. (26) which now read

$$
Q_{k}=\pi_{\omega} M_{1 k}+\pi_{\zeta} M_{2 k} .
$$

We use Eq. (118) of Appendix 2 for $M$ and find

$$
\sum_{k} Q_{k}^{2}=\pi_{\omega}^{2}+\frac{1}{\sin ^{2} \omega_{0}} \pi_{\zeta}^{2}
$$

That shows that the Hamiltonian (32) is proportional to the square of the total angular momentum,

$$
H=\frac{1}{2 C} \sum_{k} Q_{k}^{2} .
$$

In other words, we obtain the classical theory of the rotating top. The constant $C$ is the moment of inertia.

\section{Another Parameterization}

We have actually carried the approach of Section 2 further, deriving the Hamiltonian and quantizing it. The resulting equations are difficult to interpret, however. They do not display in an obvious fashion what is expected on physical grounds: Harmonic vibrational motion of the variables $\omega_{L}$ and $\zeta_{L}$. As shown in Appendix 3, these difficulties have to do with the non-Cartesian form of the measure $\mathrm{d} E$ in Eq. (21). That is why we now introduce another parameterization of the matrix $U$ defined in Eq. (1). We proceed in close analogy to Section 2 .

\subsection{Nambu-Goldstone Modes}

We use the space-fixed system, and we parameterize the matrix $U$ in product form,

$$
\begin{aligned}
& U \quad=g(\zeta, \omega) u(x, y), \\
& g(\zeta, \omega)=\exp \left\{-i \zeta(t) \hat{J}_{z}\right\} \exp \left\{-i \omega(t) \hat{J}_{y}\right\}, \\
& u(x, y)=\exp \left\{-i x \hat{J}_{x}-i y \hat{J}_{y}\right\} .
\end{aligned}
$$

The purely time-dependent variables $\omega$ and $\zeta$ describe rotations of the finite system, similarly to the variables $\omega_{0}, \zeta_{0}$ introduced in Eqs. (5). As in Section 2 we choose the ranges as $0 \leq \omega, \zeta \leq 2 \pi$. This is convenient for Section 4.1. With $\theta$ and $\phi$ as defined in Section 2, the fields $x=x(\theta, \phi, t)$ and $y=y(\theta, \phi, t)$ play the role of the fields $\omega_{1}$ and $\zeta_{1}$ defined in Eqs. (5). They describe the small-amplitude vibrations of the liquid drop. To exclude the possibility that $x$ and $y$ induce a global rotation of the entire drop we request

$$
\int \mathrm{d} E x(\theta, \phi, t)=0=\int \mathrm{d} E y(\theta, \phi, t) .
$$


We use the definition that $U=g u$ acts onto objects to the right. Thus, the local vibrations induced by the field $u$ are followed by a global rotation $g$ of the entire drop.

We show in Appendix 3 that the parameterization of $U$ in terms of the variables $\omega$ and $\zeta$ in Eqs. (44) and (5) and the one introduced in Eq. (36) are completely equivalent. Why then did we not start from the outset with the parameterization (36)? As shown in Section 2, the parameterization used in Eqs. (4) and (5) can be justified physically in a convincing manner. Moreover, it is tailored after the standard approach to symmetry breaking in non-relativistic systems. The advantage of the new parameterization is that it treats the rotational degrees of freedom separately while the parameterization in Eqs. (4) and (5) treats the rotational mode and the vibrational modes on an equal footing. It has an alternative physical interpretation. When acting from right to left, $u$ induces a small-amplitude dislocation of a nucleon (or volume element) at $(\theta, \phi)$ in the axially-symmetric nucleus (whose symmetry axis is the $z$ axis), while $g$ then rotates the entire nucleus.

Power counting as in Section 2.4 shows that

$$
\begin{aligned}
\omega, \zeta & \sim \mathcal{O}(1), \\
\dot{\omega}, \dot{\zeta} & \sim \xi, \\
|x|,|y| \sim \varepsilon^{1 / 2} \ll 1, & \\
\dot{x}, \dot{y} & \sim \Omega \varepsilon^{1 / 2} .
\end{aligned}
$$

The parameter $\varepsilon$ helps to identify (and omit) higher powers of $x$ and $y$, consistent with a focus on small-amplitude harmonic surface vibrations. A physical interpretation of this parameter is given at the end of Section 3.3 below. From here on the development is similar to that of Section 2 .

In analogy to Eq. (6) we define

$$
U^{-1} i \partial_{\mu} U=a_{\mu}^{x} J_{x}+a_{\mu}^{y} J_{y}+a_{\mu}^{z} J_{z}
$$

As in Section 2 the symbol $\partial_{\mu}$ with $\mu=1,2,3$ stands for the partial derivatives with respect to the angles $\theta, \phi$ and time $t$ while in the present Section $\partial_{\nu}$ with $\nu=1,2$ stands for the derivatives with respect to the angles only. To work out the Nambu-Goldstone modes explicitly we use

$$
\begin{aligned}
g^{-1} \partial_{t} g= & -i\left(-\dot{\zeta} \sin \omega J_{x}+\dot{\omega} J_{y}+\dot{\zeta} \cos \omega J_{z}\right), \\
u^{-1} \partial_{t} u= & -i\left(\dot{x}+\frac{y}{6}(x \dot{y}-y \dot{x})\right) J_{x} \\
& -i\left(\dot{y}-\frac{x}{6}(x \dot{y}-y \dot{x})\right) J_{y} \\
& -i\left(\frac{1}{2}(y \dot{x}-x \dot{y})\right) J_{z}
\end{aligned}
$$

and, from the Baker-Campbell-Haussdorf expansion,

$$
\begin{aligned}
& U^{-1} \partial_{\nu} U=u^{-1} \partial_{\nu} u, \\
& U^{-1} \partial_{t} U=u^{-1} \partial_{t} u+u^{-1}\left(g^{-1} \partial_{t} g\right) u
\end{aligned}
$$


Effective Field Theory of Emergent Symmetry Breaking in Deformed Atomic Nuclei 13

$$
\begin{aligned}
= & -i\left(\dot{x}-\dot{\zeta} \sin \omega+\frac{y}{6}(x \dot{y}-y \dot{x})-y \dot{\zeta} \cos \omega\right) J_{x} \\
& -i\left(\dot{y}+\dot{\omega}-\frac{x}{6}(x \dot{y}-y \dot{x})+x \dot{\zeta} \cos \omega\right) J_{y} \\
& -i\left(\frac{1}{2}(y \dot{x}-x \dot{y})+\dot{\zeta} \cos \omega-x \dot{\omega}-y \dot{\zeta} \sin \omega\right) J_{z}+\ldots .
\end{aligned}
$$

Here and in what follows, the dots indicate terms of higher order in $\varepsilon$. From Eqs. (40) and (41) we obtain

$$
\begin{aligned}
& a_{t}^{x}=\dot{x}+\frac{y}{6}(x \dot{y}-y \dot{x})-\dot{\zeta} \sin \omega-y \dot{\zeta} \cos \omega+\ldots, \\
& a_{t}^{y}=\dot{y}-\frac{x}{6}(x \dot{y}-y \dot{x})+\dot{\omega}+x \dot{\zeta} \cos \omega+\ldots, \\
& a_{t}^{z}=-\frac{1}{2}(x \dot{y}-y \dot{x})+\dot{\zeta} \cos \omega-y \dot{\zeta} \sin \omega-x \dot{\omega}+\ldots,
\end{aligned}
$$

and

$$
\begin{aligned}
a_{\nu}^{x} & =\partial_{\nu} x+\frac{y}{6}\left(x \partial_{\nu} y-y \partial_{\nu} x\right)+\ldots, \\
a_{\nu}^{y} & =\partial_{\nu} y-\frac{x}{6}\left(x \partial_{\nu} y-y \partial_{\nu} x\right)+\ldots, \\
a_{\nu}^{z} & =-\frac{1}{2}\left(x \partial_{\nu} y-y \partial_{\nu} x\right)+\ldots
\end{aligned}
$$

Eqs. (42) and (43) give the lowest-order contributions to the Nambu-Goldstone modes for the parameterization (36).

\subsection{Invariants}

As in Section 2.2 we build the effective Lagrangian upon invariants constructed from the Nambu-Goldstone modes. To this end we need to determine the behavior of these modes under transformations. We consider a rotation $r$ about infinitesimal angles $\delta \chi_{k}$ around the space-fixed $k=x, y, z$ axes. We use Eq. (36) for $U$. With

$$
r g(\zeta, \omega)=g\left(\zeta^{\prime}, \omega^{\prime}\right) h\left(\gamma^{\prime}\right), h\left(\gamma^{\prime}\right)=\exp \left\{i \gamma^{\prime} J_{z}\right\},
$$

and $\zeta^{\prime}, \omega^{\prime}, \gamma^{\prime}$ given below we have

$$
r U=r g(\zeta, \omega) u(x, y)=g\left(\zeta^{\prime}, \omega^{\prime}\right) h\left(\gamma^{\prime}\right) u=g\left(\zeta^{\prime}, \omega^{\prime}\right)\left[h\left(\gamma^{\prime}\right) u h^{\dagger}\left(\gamma^{\prime}\right)\right] h\left(\gamma^{\prime}\right)=U^{\prime} h .
$$

The last of equations (45) defines $U^{\prime} \equiv U\left(\zeta^{\prime}, \omega^{\prime}, x^{\prime}, y^{\prime}\right)$ as an element of the coset space $S O(3) / S O(2)$. We accordingly write

$$
U^{\prime-1} i \partial_{\mu} U^{\prime}=\left(a_{\mu}^{x}\right)^{\prime} J_{x}+\left(a_{\mu}^{y}\right)^{\prime} J_{y}+\left(a_{\mu}^{z}\right)^{\prime} J_{z} .
$$

Proceeding as in Section 2.2 we have

$$
\begin{aligned}
U^{-1} \partial_{\mu} U & \rightarrow(r U)^{-1} \partial_{\mu}(r U) \\
& =\left(U^{\prime} h\right)^{-1} \partial_{\mu}\left(U^{\prime} h\right) \\
& =h^{-1}\left(U^{\prime}\right)^{-1}\left(\partial_{\mu} U^{\prime}\right) h+h^{-1}\left(\partial_{\mu} h\right) .
\end{aligned}
$$


From Eqs. (47, 46) and Eq. (39) we obtain

$$
\left(\begin{array}{c}
\left(a_{\mu}^{x}\right)^{\prime} \\
\left(a_{\mu}^{y}\right)^{\prime}
\end{array}\right)=\left(\begin{array}{cc}
\cos \gamma^{\prime} & -\sin \gamma^{\prime} \\
\sin \gamma^{\prime} & \cos \gamma^{\prime}
\end{array}\right)\left(\begin{array}{c}
a_{\mu}^{x} \\
a_{\mu}^{y}
\end{array}\right)
$$

and

$$
\left(a_{\mu}^{z}\right)^{\prime}=a_{\mu}^{z}+\delta_{\mu t} \dot{\gamma}^{\prime} .
$$

We have used that $\gamma^{\prime}$ as given in Eq. (52) below is a function of $t$ only.

It remains to work out the relation between the variables $\zeta^{\prime}, \omega^{\prime}, x^{\prime}, y^{\prime}$ and $\zeta, \omega, x, y$. A calculation similar to that of Appendix 2 shows that $g(\zeta, \omega)$ transforms into $g\left(\zeta^{\prime}, \omega^{\prime}\right)$ with $\zeta^{\prime}=\zeta+\delta \zeta$ and $\omega^{\prime}=\omega+\delta \omega$ where

$$
\left(\begin{array}{l}
\delta \zeta \\
\delta \omega
\end{array}\right)=\left(\begin{array}{ccc}
-\cot \omega \cos \zeta & -\cot \omega \sin \zeta & 1 \\
-\sin \zeta & \cos \zeta & 0
\end{array}\right)\left(\begin{array}{l}
\delta \chi_{x} \\
\delta \chi_{y} \\
\delta \chi_{z}
\end{array}\right)
$$

According to Eq. (45) the matrix $u$ transforms under the action of $r$ into $h\left(\gamma^{\prime}\right) u h^{\dagger}\left(\gamma^{\prime}\right)$. That transformation differs from that of Eq. (8) because of the prefactor $g$ in the definition of $U$ in Eqs. (36). Therefore, $x$ and $y$ transform into $x^{\prime}$ and $y^{\prime}$ according to

$$
\left(\begin{array}{l}
x^{\prime} \\
y^{\prime}
\end{array}\right)=\left(\begin{array}{cc}
\cos \gamma^{\prime} & -\sin \gamma^{\prime} \\
\sin \gamma^{\prime} & \cos \gamma^{\prime}
\end{array}\right)\left(\begin{array}{l}
x \\
y
\end{array}\right)
$$

where

$$
\gamma^{\prime}=\frac{\cos \zeta}{\sin \omega} \delta \chi_{x}+\frac{\sin \zeta}{\sin \omega} \delta \chi_{y}
$$

Eq. (51) shows that $x^{2}+y^{2}$ is invariant under rotations. Moreover, since $\gamma^{\prime}$ depends on time, under rotations the four quantities $x, y, \dot{x}, \dot{y}$ are transformed into linear combinations of $x^{\prime}, y^{\prime}, \dot{x}^{\prime}, \dot{y}^{\prime}$.

We are now ready to construct the invariants. We begin with the time derivatives in Eqs. (42). Eq. (48) shows that $\left(a_{t}^{x}\right)^{2}+\left(a_{t}^{y}\right)^{2}$ is invariant. We use the power counting of Eqs. (38) (see also Section 3.4 below) and drop terms of order $\xi^{2} \varepsilon$ and $\xi \Omega \varepsilon^{k}$ with $k \geq 3 / 2$. We also omit terms linear in $x, y, \dot{x}$, or $\dot{y}$ as these vanish upon integration over $\theta$ and $\phi$, see Eqs. (37). That gives

$$
\left(a_{t}^{x}\right)^{2}+\left(a_{t}^{y}\right)^{2} \approx \dot{\omega}^{2}+\dot{\zeta}^{2} \sin ^{2} \omega+\dot{x}^{2}+\dot{y}^{2}+2(x \dot{y}-y \dot{x}) \dot{\zeta} \cos \omega-\frac{1}{3}(x \dot{y}-y \dot{x})^{2} .
$$

The invariant form (53) is the sum of three homogeneous polynomials of orders zero, two, and four, respectively, in the variables $x, y$, and their time derivatives. Under rotations, each of these is transformed into another homogeneous polynomial of the same order, see the text below Eq. (521)). Invariance of the form (53) implies that each of the said polynomials is invariant by itself. Hence the invariants are

$$
\begin{aligned}
& \mathcal{L}_{1 a}=\dot{\omega}^{2}+\dot{\zeta}^{2} \sin ^{2} \omega, \\
& \mathcal{L}_{1 b}=\dot{x}^{2}+\dot{y}^{2}+2(x \dot{y}-y \dot{x}) \dot{\zeta} \cos \omega, \\
& \mathcal{L}_{1 c}=(x \dot{y}-y \dot{x})^{2} .
\end{aligned}
$$


The additional invariant

$$
\mathcal{L}_{1 d}=\left(x^{2}+y^{2}\right)\left[\dot{x}^{2}+\dot{y}^{2}+2(x \dot{y}-y \dot{x}) \dot{\zeta} \cos \omega\right]
$$

is obtained by multiplying $\mathcal{L}_{1 b}$ with the invariant $\left(x^{2}+y^{2}\right)$. The invariant $\mathcal{L}_{1 d}$ is of the same order as $\mathcal{L}_{1 c}$.

We turn to the invariants constructed from the derivatives with respect to the angles $\theta, \phi$ in Eqs. (43). We confine ourselves to terms of up to fourth order in $x$ and $y$ and their derivatives. Eq. (48) shows that for all $\nu=\theta, \phi$ the form $\left(a_{\nu}^{x}\right)^{2}+\left(a_{\nu}^{y}\right)^{2}$ is invariant, and so are $a_{\nu}^{z}$ and $a_{\nu}^{z} a_{\nu^{\prime}}^{z}$. Forming suitable linear combinations of these and multiplying with the additional invariant $\left(x^{2}+y^{2}\right)$ we find the invariants

$$
\begin{aligned}
& \mathcal{L}_{2 a}=(\vec{L} x)^{2}+(\vec{L} y)^{2}, \\
& \mathcal{L}_{2 a^{\prime}}=\left(L_{z} x\right)^{2}+\left(L_{z} y\right)^{2}, \\
& \mathcal{L}_{2 b}=(x \vec{L} y-y \vec{L} x)^{2}, \\
& \mathcal{L}_{2 c}=\left(x^{2}+y^{2}\right)\left((\vec{L} x)^{2}+(\vec{L} y)^{2}\right) .
\end{aligned}
$$

The construction of the invariants in Eqs. (54) to (56) is based on the fact that under rotations, $u$ transforms into $h u h^{\dagger}$. This feature does not apply in the absence of any rotational motion, i.e., for $g=1$ or $\omega=0=\zeta$. It is easy to see that in that case we would have $r U=r u=u\left(x^{\prime}, y^{\prime}\right) h$ where $x^{\prime}, y^{\prime}$ are nonlinear functions of $x, y$. The argument shows why the invariants in Eqs. (54) to (56) occur specifically in the case of rotational motion but differ in cases like ferromagnetism or paramagnetism where all modes considered are true Nambu-Goldstone modes. It also shows that care is needed when considering the limit of an infinitely large moment of inertia: In the framework of the present formalism, that limit differs from the one where rotational motion is ruled out from the outset.

In the construction of the invariants, time-reversal invariance has been taken into account in the same manner as in Section 2.2. When we apply our formalism to atomic nuclei, an additional symmetry (the $\mathcal{R}$-symmetry) comes into play. That symmetry matters for the quantized version of the theory and is, therefore, deferred to Section 4.2.

\subsection{Classical Field Theory}

As in Section 2.3 the effective Lagrangian $L$ is given in terms of an arbitrary linear combination of the invariants constructed in Section 3.2 and involves eight constants $C_{i}, i=a, b, c, d$ and $D_{i}, i=a, a^{\prime}, b, c$ that must be determined by a fit to data. In obvious notation we have

$$
\begin{aligned}
L & =L_{1}+L_{2}=\int \mathrm{d} E \mathcal{L} \\
& =\int \mathrm{d} E\left(\sum_{i=a, b, c, d} \frac{C_{i}}{2} \mathcal{L}_{1 i}-\sum_{i=a, a^{\prime}, b, c} \frac{D_{i}}{2} \mathcal{L}_{2 i}\right) .
\end{aligned}
$$

The integration over angles is defined in Eq. (21). 
In slight difference to Eqs. (27) we expand the real variable $x$ in two ways, either in spherical harmonics $Y_{L \mu}=(-)^{\mu} Y_{L-\mu}^{*}$ or in terms of the real orthonormal functions

$$
Z_{L \mu} \equiv \begin{cases}\frac{1}{\sqrt{2}}\left(Y_{L \mu}+Y_{L \mu}^{*}\right), & \mu>0, \\ Y_{L 0} & \mu=0, \\ \frac{1}{i \sqrt{2}}\left(Y_{L \mu}-Y_{L \mu}^{*}\right), & \mu<0 .\end{cases}
$$

We note that the functions $Z_{L \mu}$ do not form the components of a spherical tensor. We write

$$
\begin{aligned}
x & =\sum_{L=2}^{\infty} \sum_{\mu=-L}^{L} x_{L \mu} Z_{L \mu} \\
& =\sum_{L=2}^{\infty} \sum_{\mu=-L}^{L} \tilde{x}_{L \mu} Y_{L \mu},
\end{aligned}
$$

and correspondingly for the real variables $y, \dot{x}, \dot{y}$. As in Eqs. (27), contributions with $L=0$ and $L=1$ are excluded. The coefficients $x_{l \mu}$ are real. For the complex coefficients $\tilde{x}_{L \mu}$ we have $\tilde{x}_{L \mu}^{*}=(-)^{\mu} \tilde{x}_{L-\mu}$. For every value of $L$ the coefficients $\tilde{x}_{L \mu}$ and $x_{L \mu^{\prime}}$ are linearly related in an obvious way. The first of Eqs. (59) is useful for quantization. The second is more useful when the calculation requires angular-momentum algebra.

Using the first Eq. (59) and carrying out the integration over angles, we obtain for the total Lagrangian in Eq. (57)

$$
\begin{aligned}
L & =\frac{C_{a}}{2}\left(\dot{\omega}^{2}+\dot{\zeta}^{2} \sin ^{2} \omega\right)+\frac{C_{b}}{2} \sum_{L} \sum_{\mu}\left(\dot{x}_{L \mu}^{2}+\dot{y}_{L \mu}^{2}\right) \\
& +C_{b} \dot{\zeta} \cos \omega \sum_{L} \sum_{\mu}\left(x_{L \mu} \dot{y}_{L \mu}-y_{L \mu} \dot{x}_{L \mu}\right) \\
& -\frac{1}{2} \sum_{L \mu}\left(D_{a} L(L+1)+D_{a^{\prime}} \mu^{2}\right)\left(x_{L \mu}^{2}+y_{L \mu}^{2}\right) .
\end{aligned}
$$

We have restricted ourselves to terms up to and including the orders $\mathcal{O}(\Omega), \mathcal{O}(\varepsilon \Omega)$, and $\mathcal{O}(\xi)$. The canonical momenta are

$$
p_{\omega}=\frac{\partial \mathcal{L}}{\partial \dot{\omega}}, p_{\zeta}=\frac{\partial \mathcal{L}}{\partial \dot{\zeta}}, p_{L \mu}^{x}=\frac{\partial \mathcal{L}}{\partial \dot{x}_{L \mu}}, p_{L \mu}^{y}=\frac{\partial \mathcal{L}}{\partial \dot{y}_{L \mu}} .
$$

In analogy to the first Eq. (59) we define the real function

$$
p_{x}(\theta, \phi)=\sum_{L=2}^{\infty} \sum_{\mu=-L}^{L} p_{L \mu}^{x} Z_{L \mu}(\theta, \phi),
$$

and correspondingly for $p_{y}(\theta, \phi)$.

We use the Noether theorem as in Section 2.3 and find the conserved quantities $(k=x, y, z)$

$$
Q_{k}=\int \mathrm{d} E\left\{\frac{\delta \omega}{\delta \chi_{k}} p_{\omega}+\frac{\delta \zeta}{\delta \chi_{k}} p_{\zeta}+\frac{\delta x}{\delta \chi_{k}} p_{x}+\frac{\delta y}{\delta \chi_{k}} p_{y}\right\} .
$$


We identify the $Q_{k}$ with the three components of angular momentum. Explicitly we obtain from Eq. (50) and from the differential form of Eq. (51)

$$
\begin{aligned}
& Q_{x}=-p_{\omega} \sin \zeta-p_{\zeta} \cot \omega \cos \zeta+\frac{\cos \zeta}{\sin \omega} K, \\
& Q_{y}=p_{\omega} \cos \zeta-p_{\zeta} \cot \omega \sin \zeta+\frac{\sin \zeta}{\sin \omega} K, \\
& Q_{z}=p_{\zeta} .
\end{aligned}
$$

Here

$$
K=\int \mathrm{d} E\left(x p_{y}-y p_{x}\right)
$$

The terms in Eqs. (64) that do not involve the factor $K$ correspond to the angular momentum of the rigid rotor. That is shown below and is analogous to Section 2.5. The integral $K$ over solid angle in Eq. (65) is the angular momentum of the two-dimensional oscillators that describe the surface vibrations. That is easily shown by applying the Noether theorem to $\int \mathrm{d} E\left(x^{2}+y^{2}\right)$. As remarked below Eq. (52), that expression is invariant under $S O(2)$ transformations of $(x, y)$. The conserved quantity associated with this invariance is $K$. The square of the total angular momentum is

$$
Q^{2}=p_{\omega}^{2}+\frac{1}{\sin ^{2} \omega}\left(p_{\zeta}^{2}-2 K p_{\zeta} \cos \omega+K^{2}\right)
$$

\subsection{Power Counting}

Given the full Lagrangian in Eq. (60) we can now complete the arguments leading to the relations (38). To identify the terms that are kept we use arguments similar to the ones in Section 2.4. In addition to Eqs. (38) we assume

$$
\begin{aligned}
& D_{a}, D_{a^{\prime}} \sim \Omega / \varepsilon, \\
& C_{a} \sim \xi^{-1}, \\
& C_{b}, C_{c}, C_{d} \sim(\varepsilon \Omega)^{-1},
\end{aligned}
$$

which implies

$$
\begin{array}{ll}
C_{a} \mathcal{L}_{1 a} & \sim \xi, \\
C_{b} \mathcal{L}_{1 b} & \sim \Omega, \\
C_{c} \mathcal{L}_{1 c}, C_{d} \mathcal{L}_{1 d} & \sim \varepsilon \Omega,
\end{array}
$$

and

$$
\begin{aligned}
& p_{\omega}, p_{\zeta} \sim 1, \\
& p_{x}, p_{y} \sim \varepsilon^{-1 / 2} .
\end{aligned}
$$

If we were to scale $C_{c} \sim\left(\varepsilon^{2} \Omega\right)^{-1}$ we would find $C_{c} \mathcal{L}_{1 c} \sim \Omega$, and that would be unexpected (or "unnatural") for a term with such a high power in the coordinates $x, y$. We cannot completely rule out this possibility, however, as effective field theories with unnaturally large scale do exist. As an example we mention the pion-less nuclear effective field theory 
for systems with a large scattering length [20, 21]. The large values of $p_{x}, p_{y}$ implied by the relations (69) do not seem natural but cannot be avoided if we insist on $x, y \sim \varepsilon^{1 / 2}$.

To illuminate the role of the dimensionless parameter $\varepsilon$ we consider the limit of an infinite system (with infinite moment of inertia $C_{a}$, or $\xi \rightarrow 0$ ). The fields $\zeta$ and $\omega$ become static and have constant values that depend on the orientation of the rotor. Spontaneous symmetry breaking gives rise to the Nambu-Goldstone fields $x$ and $y$ that describe the low-energy modes at the scale $\Omega$. The effective field theory breaks down at the scale $\Lambda \gg \Omega$. The assumption $\varepsilon \sim \Omega / \Lambda \ll 1$ takes account of this fact and is similarly used in other non-relativistic applications of effective field theory [5, 6]. Terms of higher order in the time derivatives of $x$ or $y$ that were suppressed in Eq. (53) would be of order $\Omega \varepsilon^{k}$ with $k \geq 3 / 2$ and, thus, of higher order in $\Omega / \Lambda$. A similar power counting for the spatial derivatives results from the replacement $\vec{L} \rightarrow \Omega \vec{L}$ as in Section 2.4.

In the opposite case where $\Lambda \rightarrow \infty$ but where $\xi \ll \Omega$ is finite (i.e., differs from zero), the terms of order $\Omega \varepsilon \propto 1 / \Lambda$ disappear. The Hamiltonian describes a rotor coupled to a set of oscillators. A problem occurs once the excitation energy is so large that the amplitudes $x, y$ are of order unity and compete with the finite rotations of the top. A distinction between the two types of modes is then no longer meaningful, and spontaneous symmetry breaking does not give an adequate description of the system.

\subsection{Effective Hamiltonian}

The effective Hamiltonian $H$ is obtained from the effective Lagrangian $L$ via a Legendre transformation. To perform that transformation we write the kinetic part $L_{1}$ of $L$ in a form that displays its bilinear dependence on the velocities. We define the infinite-dimensional velocity vector $V^{T}=\left\{\dot{\zeta}, \dot{\omega}, \dot{x}_{L \mu}, \dot{y}_{L \mu}\right\}$ where $L=2,3, \ldots$ and $\mu=L, L-1, \ldots,-L$ and write $L_{1}=(1 / 2) V^{T} \hat{G} V$. The matrix $\hat{G}$ is easily found from Eq. (60). We analogously define the vector of momenta $P^{T}=\left\{p_{\zeta}, p_{\omega}, p_{L \mu}^{x}, p_{L \mu}^{y}\right\}$. Then the effective classical Hamiltonian is

$$
H=\frac{1}{2} P^{T} \hat{G}^{-1} P+\frac{1}{2} \sum_{L \mu}\left(D_{a} L(L+1)+D_{a^{\prime}} \mu^{2}\right)\left(x_{L \mu}^{2}+y_{L \mu}^{2}\right) .
$$

To calculate the inverse $\hat{G}^{-1}$ we write $\hat{G}=\hat{G}_{0}+\hat{G}_{1}$, where $\hat{G}_{0}$ is the diagonal part of $\hat{G}$, and use perturbation theory in $\hat{G}_{1}$ so that $\hat{G}^{-1}=\hat{G}_{0}^{-1}-\hat{G}_{0}^{-1} \hat{G}_{1} \hat{G}_{0}^{-1}+\hat{G}_{0}^{-1} \hat{G}_{1} \hat{G}_{0}^{-1} \hat{G}_{1} \hat{G}_{0}^{-1} \pm$ .... Keeping only terms up to and including the orders $\mathcal{O}(\Omega), \mathcal{O}(\varepsilon \Omega)$, and $\mathcal{O}(\xi)$ we obtain

$$
\begin{aligned}
H= & \frac{1}{2} \sum_{L \mu}\left[\frac{1}{C_{b}}\left(\left(p_{L \mu}^{x}\right)^{2}+\left(p_{L \mu}^{y}\right)^{2}\right)+\left(D_{a} L(L+1)+D_{a^{\prime}} \mu^{2}\right)\left(x_{L \mu}^{2}+y_{L \mu}^{2}\right)\right] \\
& +\frac{1}{2 C_{a}}\left[p_{\omega}^{2}+\frac{1}{\sin ^{2} \omega}\left(p_{\zeta}^{2}+2 K p_{\zeta} \cos \omega+K^{2} \cos ^{2} \omega\right)\right] \\
= & \frac{1}{2} \sum_{L \mu}\left[\frac{1}{C_{b}}\left(\left(p_{L \mu}^{x}\right)^{2}+\left(p_{L \mu}^{y}\right)^{2}\right)+\left(D_{a} L(L+1)+D_{a^{\prime}} \mu^{2}\right)\left(x_{L \mu}^{2}+y_{L \mu}^{2}\right)\right] \\
& +\frac{1}{2 C_{a}}\left(Q^{2}-K^{2}\right) .
\end{aligned}
$$


We have used Eq. (66) and [see Eq. (65)]

$$
K=\sum_{L \mu}\left(x_{L \mu} p_{L \mu}^{y}-y_{L \mu} p_{L \mu}^{x}\right) .
$$

In the Hamiltonian (71), the Nambu-Goldstone modes undergo harmonic vibrations. These are coupled via $K$ to a rigid rotor. The vibrations are of order $\mathcal{O}(\Omega)$, while the rotations are of order $\mathcal{O}(\xi)$.

We mention in passing that an alternative (and simpler) form of the Legendre transformation seems to exist. Instead of defining the momenta as in Eqs. (61) and using Eq. (70), one might define the canonical momenta $p_{x}(\theta, \phi)$ and $p_{y}(\theta, \phi)$ as functional derivatives of $\sum_{i} C_{i} \mathcal{L}_{1 i}$ with respect to $x(\theta, \phi)$ and $y(\theta, \phi)$, use $\left(p_{1}, p_{2}, p_{3}\right)=\left(p_{\zeta}, p_{x}, p_{y}\right)$ and $\left(q_{1}, q_{2}, q_{3}\right)=(\zeta, x, y)$, and define $H$ as

$$
H=p_{\omega} \dot{\omega}+\int \mathrm{d} E \sum_{i=1}^{3} p_{i} \dot{q}_{i}-L .
$$

That procedure yields the same result as the one used above only to first order in $\hat{G}_{1}$. The terms of second order differ, and the Hamiltonian resulting from Eq. (73) is not rotationally invariant. More precisely: Upon quantization $H$ does not commute with the three components $Q_{k}$ of angular momentum in Eq. (64). Therefore, we have not used Eq. (173).

\section{Quantized Hamiltonian}

In the present Section we complete the program of the paper. We quantize the effective Hamiltonian (71), we discuss two important discrete symmetries, and we investigate the resulting spectra.

\subsection{Quantization}

In quantizing $H$ and the three components $Q_{k}$ of angular momentum, we encounter the problem that $\omega$ and $\zeta$ are curvilinear coordinates, and that quantization in such coordinates is ambiguous, see Ref. [22] and references therein. The quantization depends on the physical constraints that limit the dynamics to the curved manifold, and thereby on the physical situation. We focus attention on the relevant parts of $H$ and of the $Q_{k}$. These are given by the Hamiltonian $H^{\text {rot }}$ of the rigid rotor,

$$
H^{\mathrm{rot}}=\frac{1}{2 C_{a}}\left(p_{\omega}^{2}+\frac{1}{\sin ^{2} \omega} p_{\zeta}^{2}\right)
$$

and by the associated rigid-rotor parts of Eqs. (64),

$$
\begin{aligned}
& Q_{x}^{\mathrm{rot}}=-p_{\omega} \sin \zeta-p_{\zeta} \cot \omega \cos \zeta, \\
& Q_{y}^{\mathrm{rot}}=p_{\omega} \cos \zeta-p_{\zeta} \cot \omega \sin \zeta, \\
& Q_{z}^{\mathrm{rot}}=p_{\zeta} .
\end{aligned}
$$


We use the standard approach [15] to quantizing $H^{\text {rot }}$. In Appendix 4 we describe a different approach to quantization which avoids the ambiguity encountered below and yields the same result. With

$$
G^{-1}=\left(\begin{array}{cc}
1 & 0 \\
0 & 1 / \sin ^{2} \omega
\end{array}\right)
$$

we write $H^{\text {rot }}$ in matrix form,

$$
H^{\mathrm{rot}}=\frac{1}{2 C_{a}}\left(p_{\omega}, p_{\zeta}\right) G^{-1}\left(\begin{array}{c}
p_{\omega} \\
p_{\zeta}
\end{array}\right) .
$$

Quantization is achieved upon putting

$$
\begin{aligned}
\hat{H}^{\mathrm{rot}} & =\frac{1}{2 C_{a} \sqrt{\operatorname{det} G}}\left(-i \partial_{\omega},-i \partial_{\zeta}\right) G^{-1} \sqrt{\operatorname{det} G}\left(\begin{array}{c}
-i \partial_{\omega} \\
-i \partial_{\zeta}
\end{array}\right) \\
& =-\frac{1}{2 C_{a}}\left(\partial_{\omega}^{2}+\cot \omega \partial_{\omega}+\frac{1}{\sin ^{2} \omega} \partial_{\zeta}^{2}\right) .
\end{aligned}
$$

The transition from Eq. (74) to Eq. (78) is tantamount to putting

$$
\begin{aligned}
& \hat{p}_{\omega}=-i \frac{1}{\sqrt{\sin \omega}} \partial_{\omega} \sqrt{\sin \omega}, \\
& \hat{p}_{\zeta}=-i \partial_{\zeta} .
\end{aligned}
$$

The expressions (75) must be symmetrized with respect to $\zeta, p_{\zeta}$ prior to using Eqs. (79). That gives

$$
\begin{aligned}
& \hat{Q}_{x}^{\mathrm{rot}}=i \sin \zeta \partial_{\omega}+i \cot \omega \cos \zeta \partial_{\zeta}, \\
& \hat{Q}_{y}^{\mathrm{rot}}=-i \cos \zeta \partial_{\omega}+i \cot \omega \sin \zeta \partial_{\zeta}, \\
& \hat{Q}_{z}^{\mathrm{rot}}=-i \partial_{\zeta} .
\end{aligned}
$$

It is easy to check that the three components obey $\left[\hat{Q}_{x}^{\text {rot }}, \hat{Q}_{y}^{\text {rot }}\right]=i \hat{Q}_{z}^{\text {rot }}$ (cyclic). Therefore, $\hat{\mathcal{Q}}^{\text {rot }}=\left\{\hat{Q}_{x}^{\text {rot }}, \hat{Q}_{y}^{\text {rot }}, \hat{Q}_{z}^{\text {rot }}\right\}$ is a bona fide angular-momentum operator.

For the remaining variables, we impose the usual quantization conditions and choose a representation where all the $x$ 's and $y$ 's are ordinary variables so that

$$
\hat{p}_{L \mu}^{x}=-i \frac{\partial}{\partial x_{L \mu}}, \hat{p}_{L \mu}^{y}=-i \frac{\partial}{\partial y_{L \mu}} .
$$

The components of the quantized angular momentum operator are given by

$$
\begin{aligned}
& \hat{Q}_{x}=i \sin \zeta \partial_{\omega}+i \cot \omega \cos \zeta \partial_{\zeta}+\frac{\cos \zeta}{\sin \omega} \hat{K}, \\
& \hat{Q}_{y}=-i \cos \zeta \partial_{\omega}+i \cot \omega \sin \zeta \partial_{\zeta}+\frac{\sin \zeta}{\sin \omega} \hat{K}, \\
& \hat{Q}_{z}=-i \partial_{\zeta},
\end{aligned}
$$

with the operator $\hat{K}$ defined in Eq. (65) and the quantization condition (181). The $\hat{Q}_{k}$ obey the commutation relations

$$
\left[\hat{Q}_{x}, \hat{Q}_{y}\right]=i \hat{Q}_{z}(\text { cyclic }) \text {. }
$$


The square of the total angular momentum operator is

$$
\hat{\mathcal{Q}}^{2}=-\partial_{\omega}^{2}-\cot \omega \partial_{\omega}+\frac{1}{\sin ^{2} \omega}\left(-\partial_{\zeta}^{2}+2 i \hat{K} \cos \omega \partial_{\zeta}+\hat{K}^{2}\right) .
$$

It is obvious that $\left[\hat{Q}_{k}, \hat{K}\right]=0$ for $k=x, y, z$. A complete set of commuting angularmomentum operators is, thus, $\hat{\mathcal{Q}}^{2}, \hat{Q}_{z}, \hat{K}$. That is expected: For the axially symmetric rotor the square $\hat{\mathcal{Q}}^{2}$ of the total angular momentum and its projections $\hat{Q}_{z}$ onto the space-fixed and $\hat{K}$ onto the symmetry axes are constants of the motion, with quantum numbers $J(J+1), M, K$, respectively.

We expect that

$$
\left[\hat{Q}_{k}, \hat{H}\right]=0 \text { for } k=x, y, z .
$$

To prove Eqs. (85) we observe that the first line of Eq. (171) does not involve $\hat{p}_{\omega}$ or $\hat{p}_{\zeta}$. Therefore, it suffices to show that the terms in this line all commute with $\hat{K}$. That is straightforward. We conclude that $\hat{H}$ consists of two commuting parts: The square of the total angular momentum with quantum numbers $J(J+1), M, K$, and the Hamiltonian for the surface vibrations which carry the quantum number $K$. As a consequence, a rotational band occurs upon every eigenstate of the vibrational part

of $\hat{H}$. All rotational bands have the same moment of inertia. Differences arise only through terms not considered in the approximation leading to Eq. (60), see Section 4.4.

\subsection{Discrete symmetries}

Discrete symmetries may restrict the spectrum beyond the requirement imposed by time-reversal invariance. We follow Bohr and Mottelson [8, 23] and Weinberg [2].

We first consider $\mathcal{R}$-symmetry. That symmetry is realized if an axially symmetric nucleus is, in addition, symmetric under a rotation about $\pi$ around an axis perpendicular to the symmetry axis. For definiteness, we choose a rotation $r$ around the $y$ axis. We consider the product $g(\phi, \theta) r(0, \pi, 0)$ where (with operators acting to the right) the rotation $r$ is applied prior to $g$. We have

$$
\begin{aligned}
g(\phi, \theta) r(0, \pi, 0) & =r(\phi, \theta, 0) g(0, \pi) \\
& =g(\phi+\pi, \pi-\theta) h(\pi) .
\end{aligned}
$$

We note that the naive evaluation $g(\phi, \theta) r(0, \pi, 0)=g(\phi, \theta+\pi)$ would carry $\theta$ outside of its domain of definition $0 \leq \theta \leq \pi$. Eqs. (866) show that the rotational degrees of freedom $\theta$ and $\phi$ behave under $\mathcal{R}$ as a particle on the sphere under the usual parity, i.e. $(\theta, \phi) \rightarrow(\pi-\theta, \phi+\pi)$. The operation $\mathcal{R}$ acts also on the intrinsic variables $x, y$, and we have

$$
\begin{aligned}
u(x, y) r(0, \pi, 0) & =u(-\kappa \sin \psi, \kappa \cos \psi) r(0, \pi, 0) \\
& =g(\psi, \kappa) h(-\psi) r(0, \pi, 0) \\
& =g(\psi, \kappa) r(0, \pi, 0) h(\psi) \\
& =g(\psi+\pi, \pi-\kappa) h(\psi+\pi) \\
& =u(-(\pi-\kappa) \sin (\psi+\pi),(\pi-\kappa) \cos (\psi+\pi)) h(2 \psi)
\end{aligned}
$$


Effective Field Theory of Emergent Symmetry Breaking in Deformed Atomic Nuclei 22

$$
\begin{aligned}
& =u(-(\kappa-\pi) \sin \psi,(\kappa-\pi) \cos \psi) h(2 \psi) \\
& =u(-x,-y) h(2 \psi) .
\end{aligned}
$$

Here, we used Eqs. (120) that relate $g$ and $u$, and geometric considerations in going from the second to the third line, and from the sixth to the last line. Eqs. (87) show that the intrinsic variables transform under $\mathcal{R}$ as $(x, y) \rightarrow(-x,-y)$. According to Bohr and Mottelson [8, 23], eigenfunctions of the intrinsic and external variables must have the same $\mathcal{R}$ parity, i.e., both must simultaneously be either positive or negative.

We next consider ordinary parity $\mathcal{P}$. Following Weinberg (Ref. [2], Sect. 19.2), the fields $x$ and $y$ have the same parity as the generators $J_{x}$ and $J_{y}$. As components of an axial vector, $J_{x}$ and $J_{y}$ have positive parity. Thus $\mathcal{P}(x, y)=(x, y)$. That implies that all quantized modes (i.e., single excitations, double excitations ...) of the fields $x$ and $y$ have positive parity and are allowed.

\subsection{Spectra}

The quantized Hamiltonian is given by Eq. (71) with the understanding that the momenta are operators as defined in Eqs. (791) and (81). The term of leading order $(\mathcal{O}(\omega))$ is given by the first line and written as

$$
\hat{H}_{\omega}=\sum_{L \mu}\left(\frac{\left(p_{L \mu}^{x}\right)^{2}+\left(p_{L \mu}^{y}\right)^{2}}{2 C_{b}}+\frac{C_{b}}{2} \omega_{L \mu}^{2}\left(x_{L \mu}^{2}+y_{L \mu}^{2}\right)\right) .
$$

It describes an infinite set of uncoupled harmonic oscillators with frequencies $\omega_{L \mu}=$ $\left[\left(L(L+1) D_{a}+\mu^{2} D_{b}\right) / C_{b}\right]^{1 / 2}$. In practice, the breakdown scale $\Lambda$ serves as a cutoff for this sum. It is useful to combine $x_{L \mu}$ and $y_{L \mu}$ into a two-dimensional $S O(2)$-symmetric harmonic oscillator with quantum numbers $n_{L \mu}=0,1,2, \ldots, k_{L \mu}=0, \pm 1, \pm 2, \ldots$, and energies $\left(2 n_{L \mu}+\left|k_{L \mu}\right|+1\right) \omega_{L \mu}$. The intrinsic angular momentum of the oscillators is

given by the eigenvalues $K=\sum_{L \geq 2} \sum_{\mu=-L}^{L} k_{L \mu}$ of the operator $\hat{K}$, see Eq. (65). The double sum extends over occupied states only.

For the ground state all quantum numbers vanish. For the excited states, we assume $D_{b}>0$. The lowest vibrational state corresponds to the single-quantum excitation of the mode $\left(x_{20}, y_{20}\right)$. As shown in Section 4.2 the fields $x$ and $y$ have the same positive parity as the corresponding generators $J_{x}$ and $J_{y}$ in Eqs. (36). Thus, the lowest vibrational state has $|K|=1$ and negative $\mathcal{R}$-parity. That is indeed observed in linear molecules [24]. Nuclei, however, are different. Here, low-lying states are built from paired Fermions and have positive $\mathcal{R}$-parity. States with negative $\mathcal{R}$-parity correspond to pair breaking and have high excitation energies at or above the breakdown scale $\Lambda$ of the EFT. Thus, states with odd $K$ and positive parity are absent in the low-energy spectrum of nuclei. As an example we mention the absence of low-lying magnetic dipole excitations [25, 26], i.e., $K=1$ states with positive parity, in the spectra of deformed even-even nuclei [27, 28, 29].

For nuclei, two quanta need to be excited in the lowest $\left(x_{20}, y_{20}\right)$ mode, yielding a degenerate pair of states with $K=0$ and $|K|=2$. Data [27, 29] indeed show that the low-lying vibrations in even-even deformed nuclei have $K=0$ and $|K|=2$. In the 
actinides and rare-earth nuclei, these states have excitation energies of about $1 \mathrm{MeV}$, but are not degenerate, for two reasons. First, anharmonicities, i.e. higher-order corrections to the vibrational Hamiltonian (88) lift any degeneracies, see Section 4.4. Second, the proximity of the breakdown scale at $\Lambda \approx 2-3 \mathrm{MeV}$ amplifies such anharmonicities. Indeed, there are only few nuclei that exhibit two-phonon excitations on top of the one-phonon $K=0$ or $|K|=2$ vibrations, see Refs. [30, 31] for recent reviews. The impressive spectra of ${ }^{168} \mathrm{Er}$ [27] and of ${ }^{162} \mathrm{Dy}$ [29] confirm this picture. The positiveparity states in those spectra must be viewed as anharmonically distorted quantized vibrations corresponding to our Nambu-Goldstone modes. These spectra also show negative-parity states. These states cannot be understood within the EFT discussed in this paper; they can possibly be viewed as vibrations on top of the intrinsic odd-parity state with lowest energy.

The quantized version of the full Hamiltonian of Eq. (71) is

$$
\hat{H}_{\omega, \xi}=\hat{H}_{\omega}+\frac{\hat{I}^{2}-\hat{K}^{2}}{2 C_{a}} .
$$

The last term causes a rotational band to appear on top of each of the vibrational states (band heads). The eigenfunctions are Wigner $D$-functions $D_{M, K}^{I}(\alpha, \beta, 0)$ with total integer spin $I$ and projections $-I \leq M, K \leq I$ [16, 15]. The eigenvalues of $\hat{I}^{2}$ are $I(I+1)$ with $I \geq|K|$. At this order, all rotational bands have the same moment of inertia, and deviations from this picture are due to higher-order corrections, see Ref. [10] and Section 4.4 .

We conclude that the EFT predicts that in leading order, the Nambu-Goldstone modes due to emergent breaking of rotational symmetry yield a large number of harmonic vibrations. In next-to-leading order each of these serves as head of a rotational band. All bands have identical moments of inertia. Corrections of higher order considered in Section 4.4 lead to anharmonicities of the vibrational states and cause the moments of inertia to differ.

Given the close proximity of the breakdown scale in nuclei to the vibrational excitation energy, it is reasonable to consider a simpler - but still model-independent approach to deformed nuclei. That approach [15] uses an effective theory (as opposed to the effective field theory of the present paper). It combines the quantized rotations as lowest-energy excitations with the lowest vibrational modes. The latter correspond to the $K=0$ and $K=2$ modes of the present paper. Thus, the effective theory replaces the quantum fields $x$ and $y$ by their quantized modes of longest wave length. Our results show that the effective theory is based upon a solid field-theoretical foundation.

\subsection{Terms of Higher Order}

The extension of the effective field theory to higher-order terms is straightforward but tedious. The program is this. (i) Use the power counting of Section 3.4 to identify all terms that contribute to the effective Lagrangian in a given order. That includes terms with higher time derivatives. These can be treated by perturbative 
field redefinitions [32, 33. (ii) Expand the fields $x$ and $y$ into their normal modes as in Eqs. (59), and compute the Lagrangian by integration of the Lagrangian density as in Eqs. (57). The resulting expressions are (complicated) sums involving ClebschGordan coefficients. (iii) Perform the Legendre transformation to the Hamiltonian within perturbation theory to the desired order of the power counting. (iv) Quantize the Hamiltonian and compute the spectrum.

Obviously, steps (i) to (iii) are quite laborious. Furthermore, the kinetic part of the resulting Hamiltonian will have low-energy constants that are complicated linear combinations of the corresponding coefficients of the Lagrangian. The latter are not known, and the former need to be determined from data. It is, therefore, desirable to understand the transformation properties of the coordinates $x_{L \mu}$ and $y_{L \mu}$ and of the canonical momenta $p_{L \mu}^{x}$ and $p_{L \mu}^{y}$, and to directly construct the most general Hamiltonian that is invariant under rotations at a given order of the power counting.

The construction of the invariants is guided by the following observations. Eqs. (51) show that under rotations, the fields $x(\theta, \phi)$ and $y(\theta, \phi)$ transform as the $x$ and $y$ components of a two-dimensional vector. These transformation properties hold for every point $(\theta, \phi)$ on the unit sphere. Using the expansion of the second of Eqs. (59) we conclude that the complex normal modes $\tilde{x}_{L \mu}$ and $\tilde{y}_{L \mu}$ themselves, too, transform as the $x$ and $y$ components of a two-dimensional vector. The same is true for the corresponding canonical momenta (denoted by $\tilde{p}_{L \mu}^{x}$ and $\tilde{p}_{L \mu}^{y}$, respectively) as these stem from time derivatives of the field modes $\tilde{x}_{L \mu}$ and $\tilde{y}_{L \mu}$. The integration $\int d E$ over the Lagrangian density in Eq. (57) singles out scalars. For instance, the invariant that is bilinear in the momenta is

$$
\begin{aligned}
\sum_{L}\left(\tilde{p}_{L}^{x} \cdot \tilde{p}_{L}^{x}+\tilde{p}_{L}^{y} \cdot \tilde{p}_{L}^{y}\right) & =\sum_{L \mu}(-1)^{\mu}\left(\tilde{p}_{L \mu}^{x} \tilde{p}_{L-\mu}^{x}+\tilde{p}_{L \mu}^{y} \tilde{p}_{L-\mu}^{y}\right) \\
& =\sum_{L \mu}\left(\left(p_{L \mu}^{x}\right)^{2}+\left(p_{L \mu}^{y}\right)^{2}\right) .
\end{aligned}
$$

Here $\tilde{p}_{L}^{x}$ and $\tilde{p}_{L}^{y}$ denote spherical tensors of degree $L$ with components $\tilde{p}_{L \mu}^{x}$ and $\tilde{p}_{L \mu}^{y}$, respectively.

We apply these considerations first to the kinetic terms in Eqs. (54) and (55) and then to the potential terms in Eqs. (56). In calculating the kinetic part of the Hamiltonian we encounter the need to invert the generalized form of the matrix $\hat{G}$ in Eq. (170). So far we have taken into account only the terms $\mathcal{L}_{1 a}$ and $\mathcal{L}_{1 b}$ in Eqs. (154). As in Eqs. (57) we now consider the sum $\mathcal{L}_{1}$ of all four terms in Eqs. (54) and (55). The kinetic part $L_{1}$ of the effective Lagrangian is given by integration over $\mathrm{d} E$ of $\mathcal{L}_{1}$. We note that $\mathcal{L}_{1}$ is bilinear in the time derivatives of all dynamical variables. We omit the term $\dot{\omega}^{2}$ which gives a trivial contribution. Defining $\dot{x}_{j}$ as the totality of the time derivatives $\left\{\dot{\zeta}, \dot{x}_{L \mu}, \dot{y}_{L \mu}\right\}$ we proceed as in Section 3.5 and write

$$
L_{1}=\frac{1}{2} \sum_{i j} \dot{x}_{i} A_{i j} \dot{x}_{j} \text {. }
$$

In order not to overburden the notation we have chosen the letter $A$ rather than $G$ for 
the matrix defining $L_{1}$. As in Section 3.5 we write $\hat{A}$ for the matrix and $A_{i j}$ for its elements. We have $A_{i j}=A_{j i}$, and we write $\hat{A}=\hat{A}^{(0)}+\hat{A}^{(1)}$. Here $\hat{A}^{(0)}\left(\hat{A}^{(1)}\right)$ is the sum of the contributions arising from $\mathcal{L}_{1 a}$ and $\mathcal{L}_{1 b}$ (from $\mathcal{L}_{1 c}$ and $\mathcal{L}_{1 d}$, respectively). The latter are small of order $\varepsilon$ with respect to the former. The momenta are defined by $p_{i}=\sum_{j} A_{i j} \dot{x}_{j}$, and the kinetic part of the effective Hamiltonian is

$$
H_{1}=\frac{1}{2} \sum_{i j} p_{i}\left(A^{-1}\right)_{i j} p_{j} .
$$

We calculate $\hat{A}^{-1}$ perturbatively and use the explicit form of $\mathcal{L}_{1 c}$ and $\mathcal{L}_{1 d}$ in Eqs. (54) and (55). Details are given in Appendix 5. The contributions of order $\Omega \varepsilon$ to the effective Hamiltonian stemming from $\mathcal{L}_{1 c}\left(\mathcal{L}_{1 d}\right)$ are denoted by $H_{1 c}\left(H_{1 d}\right.$, respectively). We find

$$
\begin{aligned}
& H_{1 c}=-\frac{C_{c}}{2 C_{b}^{2}} \int \mathrm{d} \Omega\left(x p_{y}-y p_{x}\right)^{2}, \\
& H_{1 d}=-\frac{C_{d}}{2 C_{b}^{2}} \int \mathrm{d} \Omega\left(x^{2}+y^{2}\right)\left(p_{x}^{2}+p_{y}^{2}\right) .
\end{aligned}
$$

A contribution of order $\Omega \varepsilon$ arises also from $\mathcal{L}_{1 b}$. It is given by

$$
H_{1 b}=\frac{1}{2 C_{a}} \frac{\cos ^{2} \omega}{\sin ^{2} \omega} \int \mathrm{d} \Omega\left(x p_{y}-y p_{x}\right)^{2} .
$$

We turn to the potential terms. For the modes $x_{L \mu}$ and $y_{L \mu}$ we deal, in analogy to Eqs. (90), with the invariant $\sum_{L \mu}\left(x_{L \mu}^{2}+y_{L \mu}^{2}\right)$. In evaluating the remaining invariants in Eqs. (56) we have to deal with the angular-momentum operators $\vec{L}$ and $L_{z}$ acting on the fields $x(\theta, \phi)$ and $y(\theta, \phi)$. Denoting by $L_{\nu}$ the spherical components of $\vec{L}$, we have

$$
\begin{aligned}
\hat{L}_{\nu} x(\theta, \phi) & =\sum_{L \mu} \tilde{x}_{L \mu}\left(\hat{L}_{\nu} Y_{L \mu}(\theta, \phi)\right) \\
& =\sum_{L \mu} \tilde{x}_{L \mu} C_{1 \nu L \mu}^{L \mu+\nu} Y_{L \mu+\nu}(\theta, \phi) .
\end{aligned}
$$

Upon multiplication with $Y_{a \alpha}^{*}(\theta, \phi)$ (where $a$ and $\alpha$ are arbitrary) and integration over $\mathrm{d} E$ we find

$$
\begin{aligned}
\int \mathrm{d} E Y_{a \alpha}^{*}(\theta, \phi) \hat{L}_{\nu} x(\theta, \phi) & =\sum_{L \mu} \tilde{x}_{L \mu} C_{1 \nu L \mu}^{L \mu+\nu} \int \mathrm{d} E Y_{a \alpha}^{*}(\theta, \phi) Y_{L \mu+\nu}(\theta, \phi) \\
& =C_{1 \nu L \alpha-\nu}^{a \alpha} \tilde{x}_{a \alpha-\nu} \\
& =(-1)^{\nu} \hat{L}_{-\nu} \tilde{x}_{a \alpha} .
\end{aligned}
$$

In the last line the operator $\hat{L}_{-\nu}$ is understood to act on the spherical tensor of rank $a$ with components $\tilde{x}_{a \alpha}$. Invariants built upon the normal modes $\tilde{x}_{L \mu}$ and $\tilde{y}_{L \mu}$ are, thus, obtained by viewing these modes as components of spherical tensors of rank $L$. Scalars are constructed after acting with $\vec{L}$ onto these components. For example

$$
\begin{aligned}
\int \mathrm{d} E(\vec{L} x)^{2} & =\sum_{\nu}(-1)^{\nu} \sum_{a \alpha} \sum_{b \beta} \tilde{x}_{a \alpha} \tilde{x}_{b \beta} \int \mathrm{d} E C_{1-\nu a \alpha}^{a \alpha-\nu} C_{1+\nu b \beta}^{b \beta+\nu} Y_{a \alpha-\nu} Y_{b \beta+\nu} \\
& =\sum_{\nu}(-1)^{\nu} \sum_{a \alpha} \sum_{b \beta} \tilde{x}_{a \alpha} \tilde{x}_{b \beta}(-1)^{\alpha-\nu} C_{1-\nu a \alpha}^{a \alpha-\nu} C_{1+\nu b \beta}^{b \beta+\nu} \delta_{a}^{b} \delta_{-\alpha+\nu}^{\beta+\nu}
\end{aligned}
$$


Effective Field Theory of Emergent Symmetry Breaking in Deformed Atomic Nuclei 26

$$
\begin{aligned}
& =-\sum_{a \alpha}(-1)^{\alpha} \tilde{x}_{a \alpha} \tilde{x}_{a-\alpha} \\
& =-\tilde{x}_{L} \cdot \tilde{x}_{L} .
\end{aligned}
$$

The evaluation of the terms in Eqs. (56) is now straightforward. Upon applying the quantization rules, we obtain higher-order terms in the Hamiltonian $\hat{H}$.

The terms in Eqs. (93) and (56) do not depend on the rotational degrees of freedom $\omega$ and $\zeta$. These terms lift the degeneracies of the vibrational modes of excitation but do not affect the moment of inertia. The term in Eq. (94) depends parametrically upon $\omega$ and couples the rotational bands with the vibrational modes.

\section{Summary and Conclusions}

We have constructed the EFT for emergent symmetry breaking in deformed nuclei. In addition to the Nambu-Goldstone modes, the theory contains two additional degrees of freedom that describe rotations about the body-fixed symmetry axis. Starting from a physically intuitive and mathematically standard parameterization where rotational and vibrational degrees of freedom are treated on an equal footing, we have switched to a much more practical parameterization where the rotational degrees of freedom receive a separate treatment. The theory is characterized by three small parameters. These are (i) the ratio $\xi / \Omega$ of the energies of rotational motion $\xi$ and of vibrational motion $\Omega$, (ii) the ratio $\Omega / \Lambda$ where $\Lambda$ is the breakdown scale of the EFT (typically given by the pairing energy or the energy of single-particle excitation in the shell model), and (iii) the parameter $\varepsilon$ which characterizes deviations from harmonicity of the vibrations. In lowest order, the spectrum consists of vibrations each of which serves as head of a rotational band. The vibrations are due to the quantized Nambu-Goldstone modes that describe the emergent breaking of $\mathrm{SO}(3)$ symmetry. In leading order, the vibrational modes are degenerate, and the rotational bands all have the same moment of inertia. Terms of next order remove both degeneracies.

\section{Acknowledgments}

This material is based upon work supported in part by the U.S. Department of Energy, Office of Science, Office of Nuclear Physics, under Award Numbers DE-FG02-96ER40963 (University of Tennessee), and under contract number DEAC05-00OR22725 (Oak Ridge National Laboratory).

\section{Appendix 1: Equations of Motion in Curvilinear Coordinates}

In curvilinear coordinates the equations of motion are obtained by variation of the product of the Lagrangian density $\mathcal{L}$ and the integration measure. We demonstrate that fact for the simplest case. We consider a Lagrangian density 
$\mathcal{L}\left(\psi, \partial \psi / \partial t, \partial \psi / \partial x_{1}, \ldots, \partial \psi / \partial x_{N}\right)$ that depends on the field $\psi$ (a function of time $t$ and of $N$ Cartesian variables $\left.x_{1}, x_{2}, \ldots, x_{N}\right)$, and on the $N+1$ derivatives of the field. Standard variation of the action integral yields

$$
\int \mathrm{d} t \int \prod_{\nu=1}^{N} \mathrm{~d} x_{\nu}\left(\frac{\partial}{\partial t} \frac{\partial \mathcal{L}}{\partial\left(\partial_{t} \psi\right)}+\sum_{\mu=1}^{N} \frac{\partial}{\partial x_{\mu}} \frac{\partial \mathcal{L}}{\partial\left(\partial_{x_{\mu}} \psi\right)}+\frac{\partial \mathcal{L}}{\partial \psi}\right) \delta \psi .
$$

We introduce $N$ curvilinear coordinates $\zeta_{k}\left(x_{1}, x_{2}, \ldots, x_{N}\right)$ with $k=1, \ldots, N$ that are functions of the $N$ Cartesian coordinates $x_{\mu}$. We define the $N$-dimensional matrix

$$
M_{\mu k}=\frac{\partial x_{\mu}}{\partial \zeta_{k}} \text { with } D=\operatorname{det} M .
$$

Then

$$
\prod_{\nu=1}^{N} \mathrm{~d} x_{\nu}=D \prod_{k=1}^{N} \mathrm{~d} \zeta_{k}, \frac{\partial}{\partial x_{\mu}}=\sum_{k=1}^{N}\left(M^{-1}\right)_{k \mu} \frac{\partial}{\partial \zeta_{k}}, \frac{\partial \mathcal{L}}{\partial\left(\partial_{x_{\mu}}\right)}=\sum_{l=1}^{N} M_{\mu l} \frac{\partial \mathcal{L}}{\partial\left(\partial_{\zeta_{l}}\right)}
$$

We insert all this into expression (98) and obtain

$$
\begin{aligned}
\int \mathrm{d} t & \int \prod_{k=1}^{N} \mathrm{~d} \zeta_{k} D\left(\frac{\partial}{\partial t} \frac{\partial \mathcal{L}}{\partial\left(\partial_{t} \psi\right)}\right. \\
& \left.+\sum_{\mu=1}^{N} \sum_{l=1}^{N}\left(M^{-1}\right)_{l \mu} \frac{\partial}{\partial \zeta_{l}} \sum_{n=1}^{N} M_{\mu n} \frac{\partial \mathcal{L}}{\partial\left(\partial_{\zeta_{n}} \psi\right)}+\frac{\partial \mathcal{L}}{\partial \psi}\right) \delta \psi .
\end{aligned}
$$

The triple sum in expression (101) can be written as

$$
\sum_{l=1}^{N} \frac{\partial}{\partial \zeta_{l}} \frac{\partial \mathcal{L}}{\partial\left(\partial_{\zeta_{l}} \psi\right)}+\sum_{\mu l n=1}^{N}\left(M^{-1}\right)_{l \mu}\left\{\frac{\partial}{\partial \zeta_{l}} M_{\mu n}\right\} \frac{\partial \mathcal{L}}{\partial\left(\partial_{\zeta_{n}} \psi\right)} .
$$

The identities $\operatorname{det} \ln D=\ln$ Trace $D$ and $\partial_{\zeta_{l}} M_{\mu n}=\partial_{\zeta_{n}} M_{\mu l}$ imply that expression (102) is equal to

$$
\sum_{l=1}^{N} \frac{\partial}{\partial \zeta_{l}} \frac{\partial \mathcal{L}}{\partial\left(\partial_{\zeta_{l}} \psi\right)}+D^{-1}\left\{\sum_{l=1}^{N} \frac{\partial}{\partial \zeta_{l}} D\right\} \frac{\partial \mathcal{L}}{\partial\left(\partial_{\zeta_{n}} \psi\right)}
$$

Using all that and the independence of $D$ of $t$ and $\psi$ we rewrite expression (101) as

$$
\int \mathrm{d} t \int \prod_{k=1}^{N} \mathrm{~d} \zeta_{k}\left(\frac{\partial}{\partial t} \frac{\partial(D \mathcal{L})}{\partial\left(\partial_{t} \psi\right)}+\sum_{l=1}^{N} \frac{\partial}{\partial \zeta_{l}} \frac{\partial(D \mathcal{L})}{\partial\left(\partial_{\zeta_{l}} \psi\right)}+\frac{\partial(D \mathcal{L})}{\partial \psi}\right) \delta \psi .
$$

Comparing expression (104) with expression (98) we conclude that variation of $\mathcal{L}$ in curvilinear coordinates is tantamount to varying $D \mathcal{L}$ and otherwise treating the curvilinear coordinates like Cartesian ones. That is what we use in Section 2.3, with $D=\sin \theta$. 
Effective Field Theory of Emergent Symmetry Breaking in Deformed Atomic Nuclei 28

\section{Appendix 2: The Matrix $M$}

We calculate the matrix $M$ defined by $\delta q_{\nu}=\sum_{k} M_{\nu k} \delta \chi_{k}$. We define

$$
\begin{aligned}
& H=\omega\left[\cos \zeta P_{x^{\prime}}+\sin \zeta P_{y^{\prime}}\right], \\
& G=\omega\left[-\sin \zeta P_{x^{\prime}}+\cos \zeta P_{y^{\prime}}\right] .
\end{aligned}
$$

Then

$$
[H, G]=-i \omega^{2} P_{z^{\prime}},\left[H,-i P_{z^{\prime}}\right]=G .
$$

With $r$ given by Eq. (23) we write

$$
r \exp \{-i H\}=\exp \{-i \tilde{H}\} \exp \left\{-i \delta \xi P_{z^{\prime}}\right\} .
$$

Here

$$
\begin{aligned}
\tilde{H} & =\tilde{\omega}\left[\cos \tilde{\zeta} P_{x^{\prime}}+\sin \tilde{\zeta} P_{y^{\prime}}\right], \\
\tilde{\omega} & =\omega+\delta \omega, \\
\tilde{\zeta} & =\zeta+\delta \zeta .
\end{aligned}
$$

We calculate $\delta \omega, \delta \zeta$ and $\delta \xi$ to first order in $\delta \chi_{k}$. Keeping only terms of first order we have with $k=x^{\prime}, y^{\prime}, z^{\prime}$

$$
\sum_{k} \delta \chi_{k} P_{k}=\frac{\delta \omega}{\omega} H+\delta \xi \exp \{-i H\} P_{z^{\prime}} \exp \{i H\}+\delta \zeta X
$$

where

$$
X=\left(\sum_{k=0}^{\infty} \frac{(-i)^{k-1}}{k !} \sum_{l=0}^{k-1} H^{l} G H^{k-l-1}\right) \exp \{i H\} .
$$

Since $X$ must be of order zero in $H$ only terms with $l=k-1$ in Eq. (110) contribute. From the commutation relations (106) we have to zeroth order in $H$

$$
\begin{aligned}
& H^{l} G \rightarrow G \omega^{l} \text { for } l \text { even } \\
& H^{l} G \rightarrow-i P_{z^{\prime}} \omega^{l+1} \text { for } l \text { odd } .
\end{aligned}
$$

Thus,

$$
X=\left(-\sin \zeta P_{x^{\prime}}+\cos \zeta P_{y^{\prime}}\right) \sin \omega+P_{z^{\prime}}(\cos \omega-1) .
$$

Similarly,

$$
\begin{aligned}
& H^{l}\left(-i P_{z^{\prime}}\right) \rightarrow G \omega^{l-1} \text { for } l \text { odd }, \\
& H^{l}\left(-i P_{z^{\prime}}\right) \rightarrow-i P_{z^{\prime}} \omega^{l} \text { for } l \text { even } .
\end{aligned}
$$

Therefore,

$$
\exp \{-i H\} P_{z^{\prime}} \exp \{i H\}=P_{z^{\prime}} \cos \omega+\left(-\sin \zeta P_{x^{\prime}}+\cos \zeta P_{y^{\prime}}\right) \sin \omega .
$$

Inserting Eqs. (114) and (112) into Eq. (109) we find

$$
\begin{aligned}
\sum_{k} \delta \chi_{k} P_{k}= & \delta \omega\left(\cos \zeta P_{x^{\prime}}+\sin \zeta P_{y^{\prime}}\right)-P_{z^{\prime}} \delta \zeta \\
& +(\delta \xi+\delta \zeta)\left[P_{z^{\prime}} \cos \omega+\left(-\sin \zeta P_{x^{\prime}}+\cos \zeta P_{y^{\prime}}\right) \sin \omega\right]
\end{aligned}
$$


We equate the coefficients multiplying $P_{x^{\prime}}, P_{y^{\prime}}$, and $P_{z^{\prime}}$ on both sides of that equation. With $\delta \omega=\delta q_{1}, \delta \zeta=\delta q_{2}, \delta \xi+\delta \zeta=\delta q_{3}$ we obtain

$$
\delta \chi_{k}=\sum_{l=1}^{3}\left(M^{-1}\right)_{k l} \delta q_{l}
$$

where

$$
M^{-1}=\left(\begin{array}{ccc}
\cos \zeta & 0 & -\sin \zeta \sin \omega \\
\sin \zeta & 0 & \cos \zeta \sin \omega \\
0 & -1 & \cos \omega
\end{array}\right) .
$$

The inverse matrix is

$$
M=\left(\begin{array}{ccc}
\cos \zeta & \sin \zeta & 0 \\
-\sin \zeta \cot \omega & \cos \zeta \cot \omega & -1 \\
-\frac{\sin \zeta}{\sin \omega} & \frac{\cos \zeta}{\sin \omega} & 0
\end{array}\right)
$$

\section{Appendix 3: Equivalence of the}

\section{Parameterizations (36) and (4, 5) for $U$}

We start from Eqs. (36) and derive Eqs. (4, 5). We define $\kappa \geq 0$ and $\Psi$ by

$$
\begin{aligned}
& x=-\kappa \sin \Psi, \\
& y=\kappa \cos \Psi .
\end{aligned}
$$

We use a power-series expansion in $\kappa$, valid for $\kappa \ll 1$. We prove the equivalence only to leading order in $\kappa$. Terms of higher order can be treated analogously. In addition to Eqs. (36) we use the following definitions and identities, valid for arbitrary values of $\kappa$, of the Euler angles $\alpha, \beta, \gamma$, and of $\Psi$,

$$
\begin{array}{ll}
h(\gamma) & \equiv e^{i \gamma J_{z}}, \\
r(\alpha, \beta, \gamma) & \equiv g(\alpha, \beta) h(\gamma), \\
u(-\kappa \sin \Psi, \kappa \cos \Psi) & =g(\Psi, \kappa) h^{\dagger}(\Psi) .
\end{array}
$$

The first of Eqs. (120) defines $h(\gamma)$ for an arbitrary angle $\gamma$. The second of Eqs. (120) defines the rotation $r(\alpha, \beta, \gamma)$ and shows that in Eqs. (36) , the factor $g(\zeta, \omega)$ acts on $u$ like a rotation $r$ with third Euler angle $\gamma=0$. The third of Eqs. (120) is an identity for the function $u(x, y)$ defined in the third of Eqs. (36). That identity can easily be derived with the help of the relation $\exp \left\{-i \Psi \hat{J}_{z}\right\} \hat{J}_{y} \exp \left\{i \Psi \hat{J}_{z}\right\}=-\sin \Psi \hat{J}_{x}+\cos \Psi \hat{J}_{y}$.

We use the transformation law

$$
r(\zeta, \omega, 0) g(\Psi, \kappa)=g\left(\zeta^{\prime}, \omega^{\prime}\right) h\left(\gamma^{\prime}\right) .
$$

Here $\zeta^{\prime}, \omega^{\prime}, \gamma^{\prime}$ are functions of the angles $\zeta$ and $\omega$ and of the variables $\Psi$ and $\kappa$ and are given in Ref. [15]. We have

$$
\begin{aligned}
\cot \left(\zeta^{\prime}-\zeta\right) & =\cos \omega \cot \Psi+\cot \kappa \frac{\sin \omega}{\sin \Psi}, \\
\cos \omega^{\prime} & =\cos \kappa \cos \omega-\sin \kappa \sin \omega \cos \Psi, \\
\cot \gamma^{\prime} & =-\cos \kappa \cot \Psi-\cot \omega \frac{\sin \kappa}{\sin \Psi} .
\end{aligned}
$$


For $|\kappa| \ll 1$ that yields in leading order

$$
\begin{aligned}
\omega^{\prime} & \approx \omega+\kappa \cos \Psi \approx \omega+y, \\
\zeta^{\prime} & \approx \zeta+\frac{\kappa \sin \Psi}{\sin \omega} \approx \zeta-\frac{x}{\sin \omega}, \\
-\gamma^{\prime} & \approx \Psi-\kappa \cot \omega \sin \Psi=\Psi+x \cot \omega .
\end{aligned}
$$

Therefore,

$$
g(\zeta, \omega) g(\Psi, \kappa) h^{\dagger}(\Psi) \approx g\left(\zeta-\frac{x}{\sin \omega}, \omega+y\right) h(x \cot \omega) .
$$

We use the third of Eqs. (120) once again to write the result as

$$
\begin{aligned}
g(\zeta, \omega) g(\Psi, \kappa) h^{\dagger}(\psi) \approx u( & \left.-(\omega+y) \sin \left(\zeta-\frac{x}{\sin \omega}\right),(\omega+y) \cos \left(\zeta-\frac{x}{\sin \omega}\right)\right) \\
& \times h(\Psi) h(x \cot \omega) .
\end{aligned}
$$

We recall that $U$ is defined in the coset space SO3/SO2. Hence

$$
U \approx u\left(-(\omega+y) \sin \left(\zeta-\frac{x}{\sin \omega}\right),(\omega+y) \cos \left(\zeta-\frac{x}{\sin \omega}\right)\right) .
$$

Eq. (126) gives the connection with the parameterization of $U$ in Eqs (44) and (5) to lowest order in $x, y$. Differences in sign are due to the fact that here we work in the space-fixed system. The variables $\omega_{0}$ and $\zeta_{0}$ in Eqs. (5) are seen to correspond to $\omega$ and $\zeta$, respectively. To lowest order in $\kappa$, the variable $\omega_{1}$ corresponds to $y$ whereas the variable $\zeta_{1}$ corresponds to $-x / \sin \omega$. The occurrence of the factor $1 / \sin \omega$ in the last relation causes the difficulties in the attempt to derive the equations of harmonic motion directly from the parameterization (41) via an expansion in powers of $\omega_{1}$ and $\zeta_{1}$ and explains why we have introduced the parametrization (36). The calculation can obviously be carried to higher orders. That establishes the complete equivalence of the parameterizations of $U$ in Eqs. (36) and (4, 5).

\section{Appendix 4: Quantization in Curvilinear Coordinates}

It is instructive to use another approach to quantization which shows how the ambiguities that are associated with the prescription (79) are avoided. We assume that $\omega$ and $\zeta$ are independent so that

$$
[\omega, \zeta]=0,\left[\omega, p_{\zeta}\right]=0,\left[p_{\omega}, \zeta\right]=0,\left[p_{\omega}, p_{\zeta}\right]=0
$$

For simplicity we have suppressed the symbol ^ on the operators. It remains to determine the commutators $\left[p_{\omega}, \omega\right]$ and $\left[p_{\zeta}, \zeta\right]$. We choose a representation where $\omega$ and $\zeta$ are ordinary real variables. Quantization is subject to three requirements. (i) The expressions for $Q_{k}^{\text {rot }}$ and for $H^{\text {rot }}$ must be Hermitian. (ii) The $Q_{k}^{\text {rot }}$ must obey the standard commutation relations $\left[Q_{x}^{\text {rot }}, Q_{y}^{\text {rot }}\right]=i Q_{z}^{\text {rot }}$ (cyclic). (iii) When expressed in terms of the quantized components $Q_{k}^{\text {rot }}$ of angular momentum, the Hamiltonian of the pure rotor must be given by Eq. (35), with $C \rightarrow C_{a}$. As for point (i), Hermitecity is defined with respect to an integration measure for the variables $\omega$ and $\zeta$. The matrix $U$ 
introduced in Eqs. (36) is defined in the coset space $S O(3) / S O(2)$. That suggests using for the volume element the expression

$$
\mathrm{d} V=\mathrm{d} \omega \sin \omega \mathrm{d} \zeta
$$

An operator $\mathcal{O}$ is Hermitian if the equality

$$
\int \mathrm{d} V \Xi^{*} \mathcal{O} \Psi=\int \mathrm{d} V(\mathcal{O} \Xi)^{*} \Psi
$$

holds for any two integrable functions $\Xi(\omega, \zeta)$ and $\Psi(\omega, \zeta)$ that are periodic with period $2 \pi$ with respect to both $\omega$ and $\zeta$. (That is why we have chosen in Section 3 the ranges of integration as $0 \leq \omega_{0}, \zeta_{0} \leq 2 \pi$ ). Requirement (ii) on the commutators of the $Q_{k}^{\text {rot }}$ and the explicit form of the $Q_{k}^{\text {rot }}$ in Eqs. (75) imply

$$
\left[p_{\omega}, \omega\right]=-i,\left[p_{\zeta}, \zeta\right]=-i
$$

Eqs. (75) show that for $Q_{k}^{\text {rot }}$ to be Hermitian, $p_{\omega}$ and $p_{\zeta}$ must be Hermitian, too. As formulated in Eq. (129), that condition is consistent with Eqs. (130) if $p_{\omega}$ and $p_{\zeta}$ obey Eqs. (179). Calculating $H^{\text {rot }}$ from Eq. (35) with $C \rightarrow C_{a}$ we obtain the second of Eqs. (78). Thus, $H^{\text {rot }}$ is the quantized Hamiltonian of a rotor with $C_{a}$ the moment of inertia.

\section{Appendix 5: Kinetic Part of the Effective Hamiltonian}

We use $\hat{A}^{-1} \approx\left(\hat{A}^{(0)}\right)^{-1}-\left(\hat{A}^{(0)}\right)^{-1} \hat{A}^{(1)}\left(\hat{A}^{(0)}\right)^{-1}+\ldots$ With

$$
L_{1 b}=\sum_{L \mu}\left[\left(\dot{x}_{L \mu}\right)^{2}+\left(\dot{y}_{L \mu}\right)^{2}\right]+2 \dot{\zeta} \cos \omega \sum_{L \mu}\left[x_{L \mu} \dot{y}_{L \mu}-y_{L \mu} \dot{x}_{L \mu}\right]
$$

we have

$$
\hat{A}^{(0)}=\left(\begin{array}{ccc}
\sin ^{2} \omega & -y_{L \mu} \cos \omega & x_{L \mu} \cos \omega \\
-y_{L \mu} \cos \omega & 1 & 0 \\
x_{L \mu} \cos \omega & 0 & 1
\end{array}\right) .
$$

The non-diagonal terms $\propto x_{L \mu}, y_{L \mu}$ are of order $\varepsilon^{1 / 2}$. Therefore, we invert $\hat{A}^{(0)}$ by expanding in powers of these terms and obtain to first order

$$
\left(\hat{A}^{(0)}\right)^{-1} \approx\left(\begin{array}{ccc}
\frac{1}{\sin ^{2} \omega} & \frac{y_{L \mu} \cos \omega}{\sin ^{2} \omega} & -\frac{x_{L \mu} \cos \omega}{\sin ^{2} \omega} \\
\frac{y_{L \mu} \cos \omega}{\sin ^{2} \omega} & 1 & 0 \\
-\frac{x_{L \mu} \cos \omega}{\sin ^{2} \omega} & 0 & 1
\end{array}\right) .
$$

The matrix $\hat{A}^{(1)}$ receives contributions from both $\mathcal{L}_{1 c}$ and $\mathcal{L}_{1 d}$. The contribution from $L_{1 c}$ is proportional to

$$
\begin{aligned}
\sum_{L L^{\prime} L^{\prime \prime} L^{\prime \prime \prime} L_{1}} & {\left[\left(x_{L \mu} \dot{y}_{L^{\prime} \mu^{\prime}}-\dot{x}_{L \mu} y_{L^{\prime} \mu^{\prime}}\right)^{L_{1}}\right.} \\
& \left.\times\left(x_{L^{\prime \prime} \mu^{\prime \prime}} \dot{y}_{L^{\prime \prime \prime} \mu^{\prime \prime \prime}}-\dot{x}_{L^{\prime \prime} \mu^{\prime \prime}} y_{L^{\prime \prime \prime} \mu^{\prime \prime \prime}}\right)^{L_{1}}\right]^{0} .
\end{aligned}
$$


The upper indices on the big round and square brackets denote the total angular momentum to which the terms are coupled. For $L_{1 d}$ we have correspondingly

$$
\begin{aligned}
\sum_{L L^{\prime} L^{\prime \prime} L^{\prime \prime \prime} L_{1}} & {\left[\left(x_{L \mu} x_{L^{\prime} \mu^{\prime}}+y_{L \mu} y_{L^{\prime} \mu^{\prime}}\right)^{L_{1}}\left(\dot{x}_{L^{\prime \prime} \mu^{\prime \prime}} \dot{x}_{L^{\prime \prime \prime} \mu^{\prime \prime \prime}}+\dot{y}_{L^{\prime \prime} \mu^{\prime \prime}} \dot{y}_{L^{\prime \prime \prime} \mu^{\prime \prime \prime}}\right)^{L_{1}}\right]^{0} } \\
+2 \dot{\zeta} \cos \omega & \sum_{L L^{\prime} L^{\prime \prime} L^{\prime \prime \prime} L_{1}}\left[\left(x_{L \mu} x_{L^{\prime} \mu^{\prime}}+y_{L \mu} y_{L^{\prime} \mu^{\prime}}\right)^{L_{1}}\right. \\
& \left.\times\left(x_{L^{\prime \prime} \mu^{\prime \prime}} \dot{y}_{L^{\prime \prime \prime} \mu^{\prime \prime \prime}}-\dot{x}_{L^{\prime \prime} \mu^{\prime \prime}} y_{L^{\prime \prime \prime} \mu^{\prime \prime \prime}}\right)^{L_{1}}\right]^{0} .
\end{aligned}
$$

Both $L_{1 c}$ and $L_{1 d}$ are small. Therefore, we calculate $-\left(\hat{A}^{(0)}\right)^{-1} \hat{A}^{(1)}\left(\hat{A}^{(0)}\right)^{-1}$ to lowest order, i.e., by taking for $\left(\hat{A}^{(0)}\right)^{-1}$ only the diagonal part of the matrix on the right-hand side of Eq. (133). The resulting terms in the effective Hamiltonian are then given by

$$
\begin{aligned}
H_{1 c}=-\frac{C_{c}}{2 C_{b}^{2}} \sum_{L L^{\prime} L^{\prime \prime} L^{\prime \prime \prime} L_{1}}\left[\left(x_{L \mu} p_{L^{\prime} \mu^{\prime}}^{y}-p_{L \mu}^{x} y_{L^{\prime} \mu^{\prime}}\right)^{L_{1}}\right. \\
\left.\quad \times\left(x_{L^{\prime \prime} \mu^{\prime \prime}} p_{L^{\prime \prime \prime} \mu^{\prime \prime \prime}}^{y}-p_{L^{\prime \prime} \mu^{\prime \prime}}^{x} y_{L^{\prime \prime \prime} \mu^{\prime \prime \prime}}\right)^{L_{1}}\right]^{0}, \\
H_{1 d}=-\frac{C_{d}}{2 C_{b}^{2}} \sum_{L L^{\prime} L^{\prime \prime} L^{\prime \prime \prime} L_{1}}\left[\left(x_{L \mu} x_{L^{\prime} \mu^{\prime}}+y_{L \mu} y_{L^{\prime} \mu^{\prime}}\right)^{L_{1}}\right. \\
\left.\quad \times\left(p_{L^{\prime \prime} \mu^{\prime \prime}}^{x} p_{L^{\prime \prime \prime} \mu^{\prime \prime \prime}}^{x}+p_{L^{\prime \prime} \mu^{\prime \prime}}^{y} p_{L^{\prime \prime \prime} \mu^{\prime \prime \prime}}^{y}\right)^{L_{1}}\right]^{0} \\
-\frac{C_{d}}{2 C_{a} C_{b}} \frac{2 p_{\zeta} \cos \omega}{\sin ^{2} \omega} \sum_{L L^{\prime} L^{\prime \prime} L^{\prime \prime \prime} L_{1}}\left[\left(x_{L \mu} x_{L^{\prime} \mu^{\prime}}+y_{L \mu} y_{L^{\prime} \mu^{\prime}}\right)^{L_{1}}\right. \\
\left.\quad \times\left(x_{L^{\prime \prime} \mu^{\prime \prime}} p_{L^{\prime \prime \prime} \mu^{\prime \prime \prime}}^{y}-p_{L^{\prime \prime} \mu^{\prime \prime}}^{x} y_{L^{\prime \prime \prime} \mu^{\prime \prime \prime}}\right)^{L_{1}}\right]^{0} .
\end{aligned}
$$

The terms inversely proportional to $C_{b}^{2}$ are of order $\varepsilon \Omega$, the term inversely proportional to $C_{a} C_{b}$ is of order $\varepsilon^{2} \Omega$ and, therefore, negligible. That result justifies a posteriori the diagonal approximation for $\left(\hat{A}^{(0)}\right)^{-1}$. With the help of the expansions (59) and (62), we obtain Eqs. (93).

\section{References}

[1] Leutwyler H 1994 Phys. Rev. D 49 3033-3043 URL http://link.aps.org/doi/10.1103/PhysRevD .49.3033

[2] Weinberg S 1996 The Quantum Theory of Fields vol II (Cambridge, UK: Cambridge University Press)

[3] Brauner T 2010 Symmetry 2 609-657 URL http://www.mdpi.com/2073-8994/2/2/609

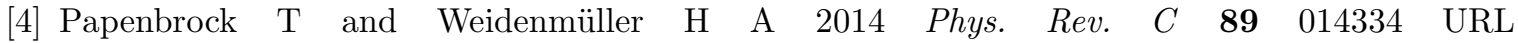
http://link.aps.org/doi/10.1103/PhysRevC.89.014334

[5] Román J M and Soto J 1999 International Journal of Modern Physics B 13 755-789 URL http://www.worldscientific.com/doi/abs/10.1142/S0217979299000655

[6] Bär O, Imboden M and Wiese U J 2004 Nuclear Physics B 686347 - 376 URL http://www.sciencedirect.com/science/article/pii/S0550321304001221

[7] Yannouleas C and Landman U 2007 Reports on Progress in Physics $\mathbf{7 0} 2067$ URL http://stacks.iop.org/0034-4885/70/i=12/a=R02 
[8] Bohr A and Mottelson B R 1975 Nuclear Structure vol II: Nuclear Deformation (Reading, Massachusetts, USA: W.A. Benjamin Inc.)

[9] Iachello F and Arima A 1987 The Interacting Boson Model (Cambridge, UK: Cambridge University Press)

[10] Zhang $\mathrm{J}$ and Papenbrock $\mathrm{T} \quad 2013$ Phys. Rev. $C^{\mathbf{8 7}} \quad 034323$ URL http://link.aps.org/doi/10.1103/PhysRevC.87.034323

[11] Coello Pérez E A and Papenbrock T 2015 ArXiv e-prints 1502.04405 URL http://adsabs.harvard.edu/abs/2015arXiv150204405C

[12] Caprio M, Maris P and Vary J 2013 Physics Letters B 719179 - 184 ISSN 0370-2693 URL http://www.sciencedirect.com/science/article/pii/S0370269313000026

[13] Dytrych T, Launey K D, Draayer J P, Maris P, Vary J P, Saule E, Catalyurek U, Sosonkina M, Langr D and Caprio M A 2013 Phys. Rev. Lett. 111252501 URL http://link.aps.org/doi/10.1103/PhysRevLett.111.252501

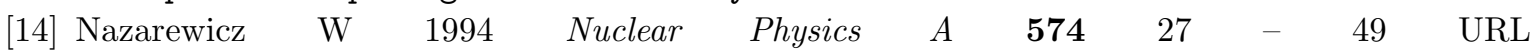
http://www.sciencedirect.com/science/article/pii/037594749490037X

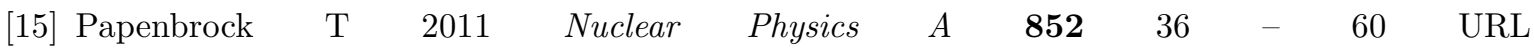
http://www.sciencedirect.com/science/article/pii/S0375947410007773

[16] Varshalovich D A, Moskalev A N and Khersonskii V K 1988 Quantum theory of angular momentum (Singapore: World Scientific)

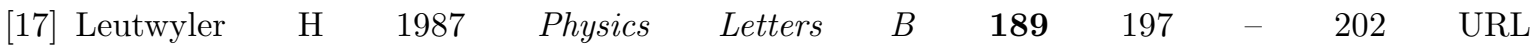
http://www.sciencedirect.com/science/article/pii/0370269387912962

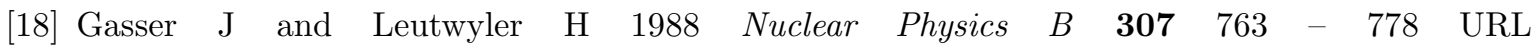
http://www.sciencedirect.com/science/article/pii/0550321388901071

[19] Hasenfratz P and Niedermayer F 1993 Zeitschrift für Physik B Condensed Matter 92 91-112 URL http://dx.doi.org/10.1007/BF01309171

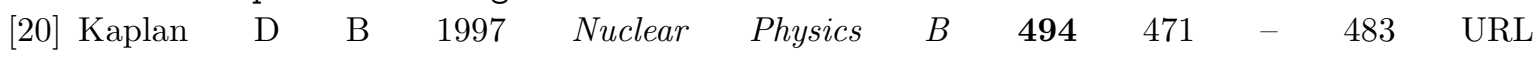
http://www.sciencedirect.com/science/article/pii/S0550321397001788

[21] Kaplan D B, Savage M J and Wise M B 1998 Nuclear Physics B 534329 - 355 URL http://www.sciencedirect.com/science/article/pii/S0550321398004404

[22] Kaplan L, Maitra N T and Heller E J 1997 Phys. Rev. A 56 2592-2599 URL http://link.aps.org/doi/10.1103/PhysRevA.56.2592

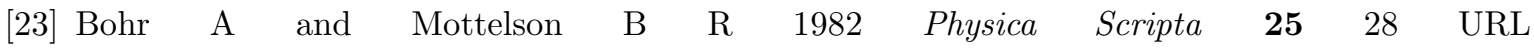
http://stacks.iop.org/1402-4896/25/i=1A/a=005

[24] Herzberg G 1945 Molecular spectra and molecular structure vol II: Infrared and Raman spectra of polyatomic molecules (New York: Van Nostrand)

[25] Heyde K, von Neumann-Cosel P and Richter A 2010 Rev. Mod. Phys. 82 2365-2419 URL http://link.aps.org/doi/10.1103/RevModPhys.82.2365

[26] Bentz W, Arima A, Enders J, Richter A and Wambach J 2011 Phys. Rev. C 84014327 URL http://link.aps.org/doi/10.1103/PhysRevC.84.014327

[27] Davidson W F, Warner D D, Casten R F, Schreckenbach K, Börner H G, Simic J, Stojanovic M, Bogdanovic M, Koicki S, Gelletly W, Orr G B and Stelts M L 1981 Journal of Physics G: Nuclear Physics 7455 URL http://stacks.iop.org/0305-4616/7/i=4/a=011

[28] Davidson W F, Warner D D, Casten R F, Schreckenbach K, Börner H G, Simic J, Stojanovic M, Bogdanovic M, Koicki S, Gelletly W, Orr G B and Stelts M L 1981 Journal of Physics G: Nuclear Physics 7843 URL http://stacks.iop.org/0305-4616/7/i=6/a=516

[29] Aprahamian A, Wu X, Lesher S R, Warner D D, Gelletly W, Börner H G, Hoyler F, Schreckenbach K, Casten R F, Shi Z R, Kusnezov D, Ibrahim M, Macchiavelli A O, Brinkman M A and Becker J A 2006 Nuclear Physics A $\mathbf{7 6 4} 42$ - 78 URL http://www.sciencedirect.com/science/article/pii/S037594740501136X

[30] Sood P, Headly D and Sheline R 1991 Atomic Data and Nuclear Data Tables 4789 - 173 URL http://www.sciencedirect.com/science/article/pii/0092640X9190019Z 
Effective Field Theory of Emergent Symmetry Breaking in Deformed Atomic Nuclei 34

[31] Sood P, Headly D and Sheline R 1992 Atomic Data and Nuclear Data Tables 51273 - 320 URL http://www.sciencedirect.com/science/article/pii/0092640X9290003Z

[32] Damour T and Schäfer G 1991 Journal of Mathematical Physics 32 127-134 URL http://link.aip.org/link/?JMP/32/127/1

[33] Grosse-Knetter C 1994 Phys. Rev. D http://link.aps.org/doi/10.1103/PhysRevD.49.6709

$49 \quad 6709-6719 \quad$ URL 